ARTICLE

\title{
The Botrytis cinerea Crh1 transglycosylase is a cytoplasmic effector triggering plant cell death and defense response
}

Kai $\mathrm{Bi}^{1,2}$, Loredana Scalschi ${ }^{1,3}$, Namrata Jaiswal $^{4}$, Tesfaye Mengiste ${ }^{4}$, Renana Fried ${ }^{1}$, Ana Belén Sanz ${ }^{5}$,

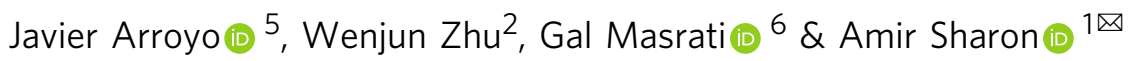

Crh proteins catalyze crosslinking of chitin and glucan polymers in fungal cell walls. Here, we show that the $\mathrm{BcCrh1}$ protein from the phytopathogenic fungus Botrytis cinerea acts as a cytoplasmic effector and elicitor of plant defense. BcCrh1 is localized in vacuoles and the endoplasmic reticulum during saprophytic growth. However, upon plant infection, the protein accumulates in infection cushions; it is then secreted to the apoplast and translocated into plant cells, where it induces cell death and defense responses. Two regions of 53 and 35 amino acids are sufficient for protein uptake and cell death induction, respectively. BcCrh1 mutant variants that are unable to dimerize lack transglycosylation activity, but are still able to induce plant cell death. Furthermore, Arabidopsis lines expressing the bccrh1 gene exhibit reduced sensitivity to $B$. cinerea, suggesting a potential use of the BcCrh1 protein in plant immunization against this necrotrophic pathogen.

\footnotetext{
${ }^{1}$ School of Plant Sciences and Food Security, Faculty of Life Sciences, Tel Aviv University, Tel Aviv, Israel. ${ }^{2}$ College of Life Science and Technology, Wuhan Polytechnic University, Wuhan City, Hubei Province, China. ${ }^{3}$ Plant Physiology Area, Biochemistry and Biotechnology Group, Department CAMN, University Jaume I, Castellón, Spain. ${ }^{4}$ Department of Botany and Plant Pathology, College of Agriculture, Purdue University, West Lafayette, IN, USA. ${ }^{5}$ Dpto. Microbiología y Parasitología, Facultad de Farmacia, Universidad Complutense, IRYCIS, Madrid, Spain. ${ }^{6}$ School of Neurobiology, Biochemistry and Biophysics, Faculty of Life Sciences, Tel Aviv University, Tel Aviv, Israel. ${ }^{凶}$ email: amirsh@tauex.tau.ac.il
} 
$B$ otrytis cinerea is a wide host-range necrotrophic plant pathogen that causes severe damages to crops worldwide ${ }^{1,2}$. The infection process includes an early stage, characterized by the formation of local necrotic lesions, an intermediate stage during which the lesions begin to spread at an increasing rate, and a late stage of constant lesion spreading ${ }^{3}$. A working model derived from these findings predicts secretion of cell death-inducing factors during the early stage that promote the formation of patches of dead tissue, which serve as foci for the subsequent stages ${ }^{4}$. This model has been supported by the discovery of secreted proteins with cell death-inducing activity, which are collectively referred to as necrosis-inducing proteins (NIPs) ${ }^{5}$. Broadly, all NIPs can be divided into two main classes: proteins that lack a known domain, and secreted enzymes that also induce plant cell death (henceforth referred to as catalytic NIPs).

The best-studied NIPs are a family of non-catalytic NIPs collectively named NEP or NELP/NLP (NEP-like proteins) ${ }^{6,7}$. Proteins in this family induce hypersensitive-like cell death in a variety of dicotyledonous, but not monocotyledonous plants ${ }^{8-10}$. Catalytic NIPs are usually glycosyl hydrolases (GHs) that degrade plant cell wall sugar polymers such as pectin ${ }^{11}$, hemicellulose $5,12,13$, and glucose polymers ${ }^{14}$. The GH NIPs that have been characterized so far remain in the apoplast after secretion by the fungus, and their cell death-inducing activity is mediated by plant extracellular membrane components, commonly in an SOBIR-BAK1-depedent manner $5,12,13$. Similar to the non-catalytic NIPs, hydrolase NIPs also induce plant defense, which in many cases was found to be unrelated to their catalytic activity ${ }^{5,12-14}$. In some instances, a short NIP-derived peptide fragment was found to be sufficient to induce necrosis and activate defense $\mathrm{e}^{13,15,16}$. In other cases, disruption of the tertiary structure of the protein eliminated necrosis- and defenseinducing activities ${ }^{5,17}$.

In search of novel NIPs, we have analyzed secretome that was collected from bean leaves after infection with $B$. cinerea spore suspension ${ }^{5}$. Here we report on the identification and characterization of BcCrh1, a GH16 transglycosylase that induces cell death and defense responses in plants. Crh (Congo red hypersensitivity) is a highly conserved family of proteins responsible for the cross-linking between chitin and glucan polymers in the fungal cell wall ${ }^{18-20}$. Hence, unlike other catalytic NIPs, BcCrh1 is not involved in plant cell wall degradation, but rather in fungal cell wall biosynthesis. Furthermore, we show that the BcCrh1 protein is internalized into the plant cell. This internalization is required for induction of cell death, meaning that BcCrh1 is a cytoplasmic effector, unlike most other NIPs that are apoplastic. We also found that BcCrh1 forms dimers, which seem to be necessary for the transglycosylase activity, while the monomeric protein is sufficient for induction of necrosis. Collectively, our study reveals an unexpected role for Crh proteins as mediators of fungal-plant interaction, and provides details on their role in fungal cell wall biosynthesis.

\section{Results}

Proteomic analysis of $B$. cinerea secretome collected from infected leaves 28 hpi revealed the presence of 259 predicted proteins ${ }^{5}$. In search of cell death-inducing proteins, we cloned selected candidate genes and transiently expressed them in Nicotiana benthamiana leaves using Agrobacterium tumefaciens-mediated transformation (Agroinfiltration assay). Bcin01g06010, which induced strong cell death in $N$. benthamiana, was further characterized.

Bcin01g06010 is a Crh family protein. Sequence analysis of the Bcin01g06010 protein using SignalP-5.0 (http://www.cbs.dtu.dk/ services/SignalP/) and TMHMM version 2.0 (http://www.cbs.dtu. $\mathrm{dk} /$ services/TMHMM/) predicted a secretion signal at the $\mathrm{N}$ terminal end and an absence of transmembrane helices, indicating that Bcin01g06010 is a secreted protein, in accordance with its presence in $B$. cinerea secretomes ${ }^{5,21,22}$. SMART (http://smart. embl.de/) analysis showed the presence of a conserved GH family 16 (GH16) domain between amino acid residues 49-263 (E-value $1.5 \mathrm{e}-36)$. A BLAST search revealed similarity to proteins in the Crh family (Supplementary Fig. 1), and 3D structure prediction showed strong structural similarity (probability 100\%, E-value 1.1e-32) to A. fumigatus AfCrh5 (Supplementary Fig. 2a). The protein was named BcCrh1 based on the homology to the Saccharomyces cerevisiae Crh1 protein. A BLAST search of the NCBI database and published RNA sequencing data ${ }^{23}$ revealed three additional B. cinerea Crh protein members that were named BcCrh2 (Bcin15g03070), BcCrh3 (Bcin13g03640) and BcCrh4 (Bcin07g04870). All four B. cinerea Crh proteins had a predicted secretion signal and a conserved GH16 domain (Supplementary Fig. 1a). Sequence alignment of the four $B$. cinerea Crh proteins with S. cerevisiae, Candida albicans, and A. fumigatus Crh homologues revealed the presence of the DEXDXE (enzymatic activity site) and the GTIXWXGG (the acceptor sugar binding site) motifs, which are highly conserved in all members of the Crh protein family (Supplementary Fig. 1b, c).

BcCrh1 cell death-inducing activity is independent of its transglycosylase activity. Agroinfiltration of $N$. benthamiana leaves with $35 \mathrm{~S}: \mathrm{BcC}$ rh1 triggered local cell death within five days post inoculation (Supplementary Fig. 3a). Infiltration assay with different concentrations of purified protein solution showed that $10 \mu \mathrm{M}$ of BcCrh1 was sufficient to cause cell death in $N$. benthamiana and tomato leaves, but not in A. thaliana, and similar to other $B$. cinerea NIPs, BcCrh1 did not promote necrosis in monocots (Supplementary Fig. 4). To test whether the GH enzymatic activity of BcCrh1 was necessary for cell deathinducing activity, we mutated the conserved residues (MBcCrh1: E120Q/D122H/E124Q) at the putative catalytic site. Agroinfiltration or injection of leaves with the MBcCrh1 both produced similar necrotic spots as infiltration with the native BcCrh1 protein (Supplementary Figs. 3a, 4, 5), indicating that induction of plant cell death is unrelated to the enzymatic activity of BcCrh1.

To verify the hypothetical transglycosylase activity of BcCrh1, we conducted a complementation assay of a $S$. cerevisiae crh $1 \Delta$ $\operatorname{crh} 2 \Delta$ double mutant strain. This strain, lacking any chitin-glucan transglycosylase activity, is hypersensitive to Congo red. Complementation of this mutant with the bccrh1 gene fully restored the wild-type phenotype (Supplementary Fig. 6). In contrast, yeast strains expressing bccrh1 with mutations in the putative catalytic residues (MBcCrh1) retained the Congo red hypersensitive phenotype. These results confirmed that BcCrh1 has a transglycosylase activity similar to the S. cerevisiae Crh1 and Crh2 proteins and that catalytic residues E120, D122, and E124 are essential for this activity.

BcCrh1 is a cytoplasmic effector. To determine the site of interaction with the plant, we performed an Agroinfiltration assay of $N$. benthamiana leaves. Two types of constructs were used, for expression of the native and enzymatic inactive proteins with (SP (PR3)-BcCrh ${ }^{121-391}$ and SP(PR3)-MBcCrh ${ }^{121-391}$ ) and without (BcCrh ${ }^{121-391}$ and $\left.\mathrm{MBcCrh}{ }^{121-391}\right)$ the A. thaliana pathogenesisrelated protein $3(\mathrm{PR} 3)^{5}$ secretion signal.

The first and second sets of constructs result in the secretion of the protein outside the plant cell, or intracellular protein accumulation, respectively. Transfection of $N$. benthamiana leaves with Agrobacterium expressing any of the four constructs 

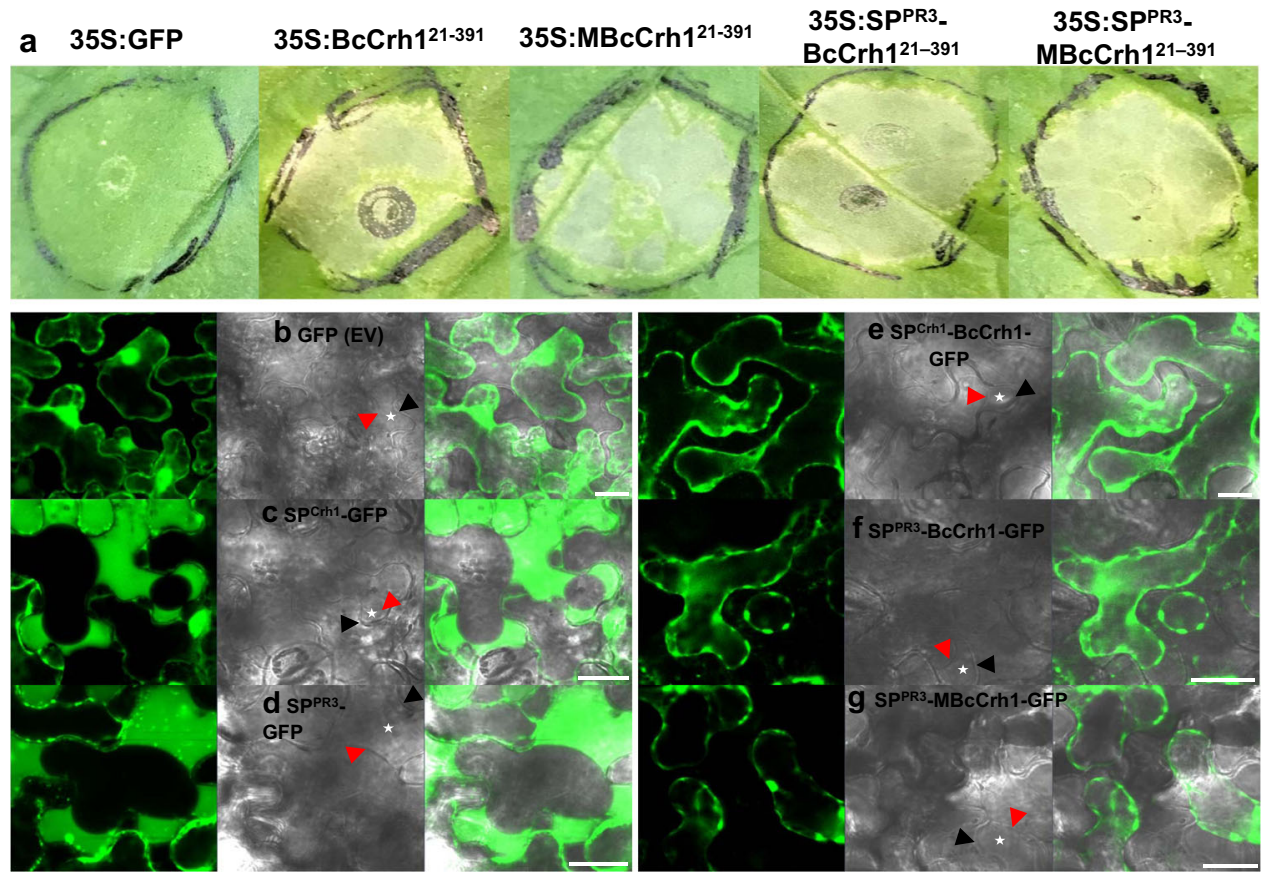

Fig. 1 Localization of BcCrh1 inside plant cells is required for induction of cell death. a Plants were infiltrated with Agrobacterium strains that were transformed with the bccrh1 gene with or without secretion signal (SP). Images of necrotic lesions (a) were taken five days after Agroinfiltration. 35S:GFP: free GFP (control); 35S:BcCrh1-SP and 35S:MBcCrh1-SP: native and enzyme inactive BcCrh1 respectively, without secretion signal; 35S:SPPR3-BcCrh121-391 and 35S:SPPR3-MBcCrh121-391: fusion of the native and enzyme inactive BcCrh1 respectively, with PR3 plant signal peptide; b-g Subcellular localization of GFP-fusion proteins. Leaves were harvested two days after Agroinfiltration, submerged for $20 \mathrm{~min}$ in $0.8 \mathrm{M}$ mannitol to induce plasmolysis, and then samples were scanned by a confocal microscope. White asterisks mark apoplastic space between the cell wall (black arrow) and plasma membrane (red arrow) in plasmolysed plant cells. Left panel shows images of cells following Agroinfiltration with free GFP (control), right panel shows images following Agroinfiltration with BcCrh1-GFP fusion protein. b GFP without SP; c GFP fused to the BcCrh1 SP; d GFP fused to PR3 SP; e BcCrh1-GFP with native SP; f BcCrh1-GFP fused to PR3 SP; $\mathbf{g}$ MBcCrh1-GFP fused to PR3 SP. Bars $=20 \mu \mathrm{m}$. All the above experiments were repeated at least three times with similar results.

induced similar cell death levels (Fig. 1a, Supplementary Fig. 5), suggesting that cytoplasmic localized $\mathrm{BcCrh} 1$ or $\mathrm{MBcC}$ rh1 induce plant cell death. To further investigate the localization of secreted BcCrh1 in plant tissues, we infiltrated plant leaves with Agrobacterium expressing a BcCrh1/MBcCrh1-GFP fusion protein with a secretion signal. Confocal fluorescence microscopy showed that the fusion protein accumulated inside the plant cells, in contrast to control leaves that were treated with Agrobacterium expressing free GFP in which the fluorescent signal was observed exclusively in the apoplastic space (Fig. 1b-g). These results confirmed that the localization of BcCrh1 is inside plant cells.

A 35-residue region of $\mathrm{BcCrh1}$ is sufficient for the plant cell death-inducing activity. To define the minimal region required for the cell death-inducing activity of $\mathrm{BcCrh} 1$, we generated a series of $\mathrm{N}$-terminal and $\mathrm{C}$-terminal deletions and tested their cell death-inducing activity. Long deletions at the $\mathrm{C}^{\prime}$ end, including $\mathrm{BcCrh}^{11-284}, \mathrm{BcCrh}^{11-214}$, and $\mathrm{BcCrh}{ }^{11-144}$, retained full activity. However, when residues $75-284$ were deleted, with ( $\left.\mathrm{BcCrh}^{11-74}\right)$ or without ( $\left.\mathrm{BcCrh}{ }^{121-74}\right)$ the secretion signal, cell death-inducing activity was lost (Fig. 2a, b). These results indicated that residues 75-144 are necessary for cell death induction. Transient expression of $\mathrm{BcCrh} 1^{75-144}$, but not $\mathrm{BcCrh} 1^{145-391}$, induced cell death in $N$. benthamiana leaves, confirming that the region between amino acids $75-144$ is both necessary and sufficient for induction of plant cell death. When $\mathrm{BcCrh} 1^{75-144}$ was fused to the $\mathrm{PR} 3$ secretion signal ( $\left.\mathrm{SP}^{\mathrm{PR} 3}-\mathrm{BcCrh} 1^{75-144}\right)$, the cell death activity was lost (Fig. 2a, b). Confocal images of $N$. benthamiana cells transiently expressing $\mathrm{BcCrh} 1^{75-144}$ or $\mathrm{SP}^{\mathrm{PR} 3}-\mathrm{BcCrh} 1^{75-144} \mathrm{GFP}$ fusions, confirmed intracellular and apoplastic localization of the proteins, respectively (Fig. 2c). Following further analyses of different parts of the protein, the region necessary for induction of cell death was narrowed down to $35 \mathrm{aa}$ between residues 93-127 (Fig. 2a, b). Since BcCrh1 ${ }^{93-127}$ contains the catalytic pocket, we mutated the catalytic residues (E120Q/D122H/E124Q) in BcCrh1 ${ }^{93-127}$. The mutated epitope (MBcCrh1 ${ }^{93-127}$ ) still triggered cell death (Fig. 2b), verifying the earlier results obtained with MBcCrh1.

A 53-residue region mediates uptake of $\mathrm{BcCrh} 1$ by plant cells. In search of specific parts of BcCrh1 that might mediate protein uptake by plant cells, we performed Agroinfiltration assays of $N$. benthamiana leaves with constructs containing the secretion sequence and various segments of the protein. The expectation was that the protein segments containing the uptake signal would first be secreted out of the plant cell and then induce cell death following processing of the secretion signal and uptake by the plant cells (as in Fig. 1). No necrosis was expected by segments lacking the uptake signal. These analyses revealed that deletion of amino acids $21-74$ ( $\left.\mathrm{BcCrh} 1^{21-74}\right)$ prevented the induction of necrosis (Fig. 2a, b), suggesting that the first 53 aa following the secretion signal mediate uptake of the $\mathrm{BcCrh} 1$ protein by plant cells. To verify this result, we fused the $\mathrm{BcCrh} 1^{1-74}$ (includes the secretion signal and the putative uptake signal) to the apoplastic NIP BcXYG1 ${ }^{5}$. Agroinfiltration with this construct failed to induce cell death, unlike control treatment with the native BcXYG1, which induced strong necrosis at 3 dpi (Fig. 3a). Consistent with the Agroinfiltration result, infiltration of tomato 
a

BcCrh11-39

BcCrh11-284

BcCrh11-214

BcCrh11-144

BcCrh1 1-74

BcCrh121-74

BcCrh175-144

BcCrh1 145-391

$\mathrm{SP}^{\mathrm{PR} 3 \mathrm{BCCrh}} 1^{75-144}$

BcCrh175-109

BcCrh1 ${ }^{110-144}$

BcCrh1 $^{\text {93-127 }}$

BcCrh1 ${ }^{93-113}$
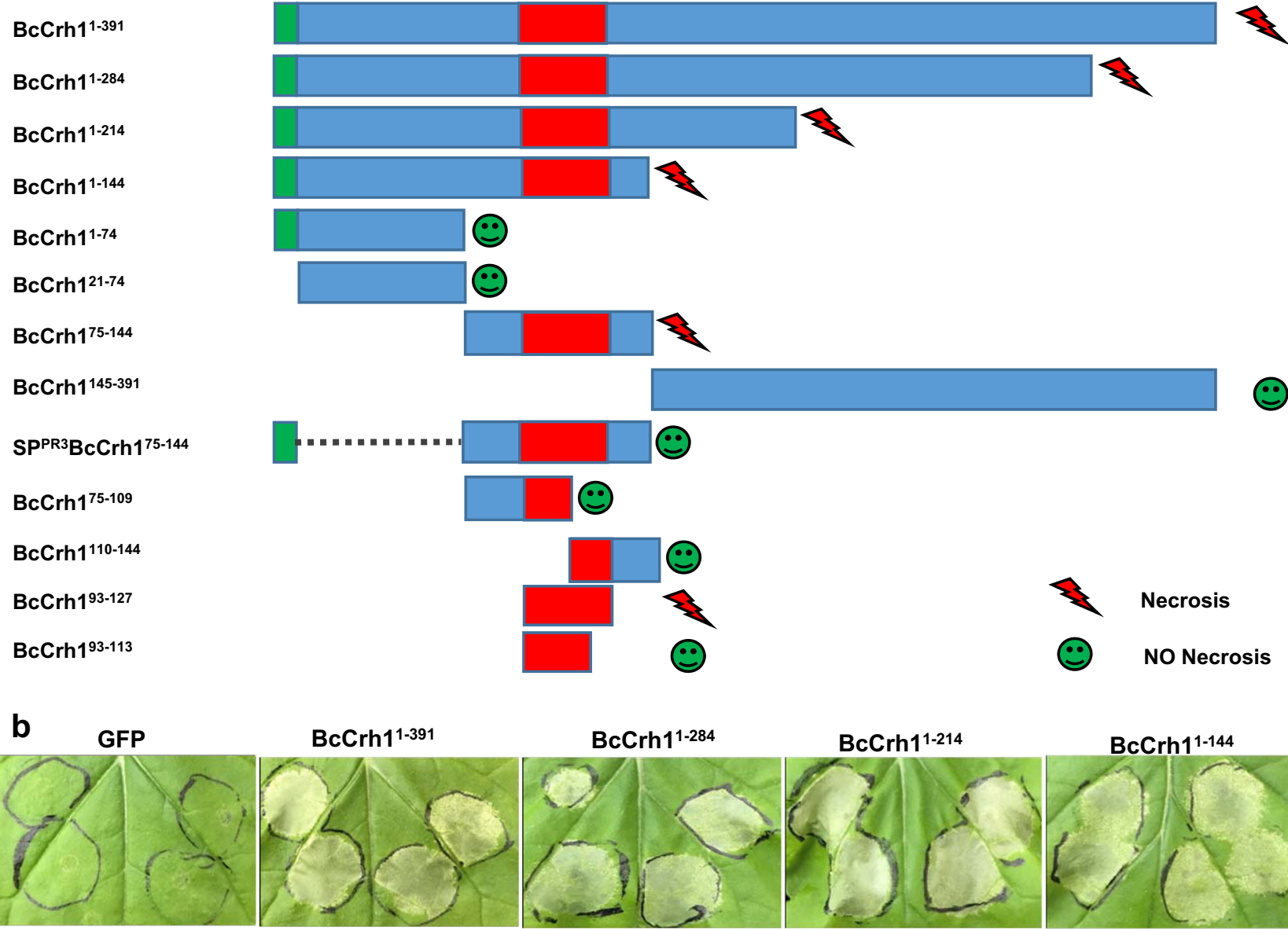

BcCrh11-214
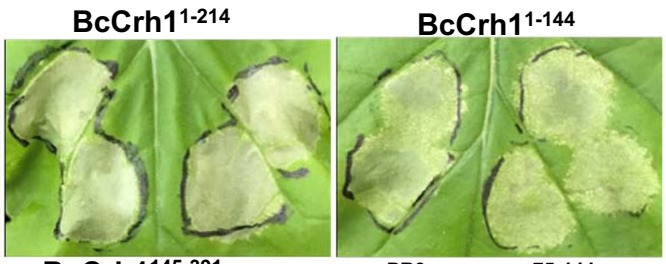

BcCrh11-74

BcCrh121-74

BcCrh175-144

BcCrh1 145-391
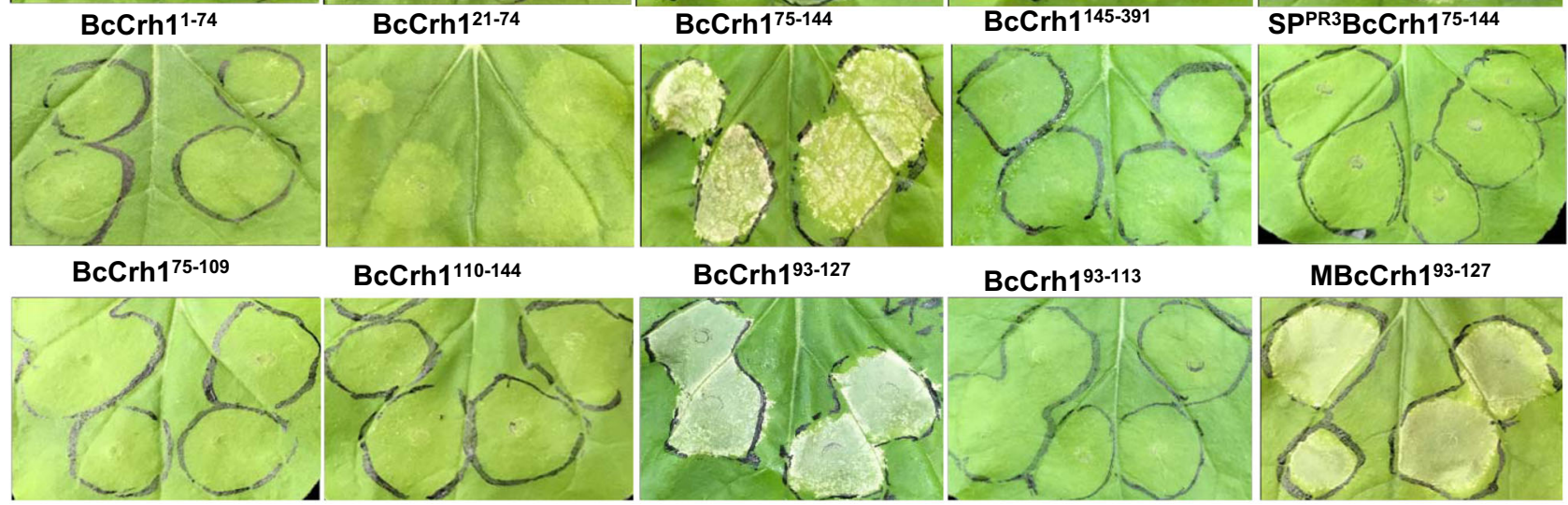

BcCrh1 110-144

BcCrh193-127
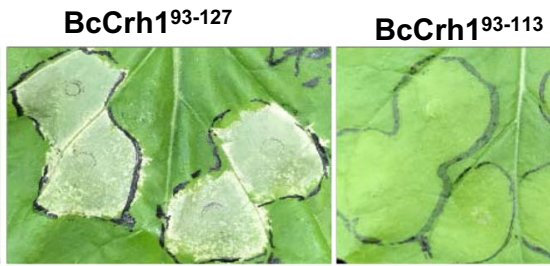

MBcCrh193-127
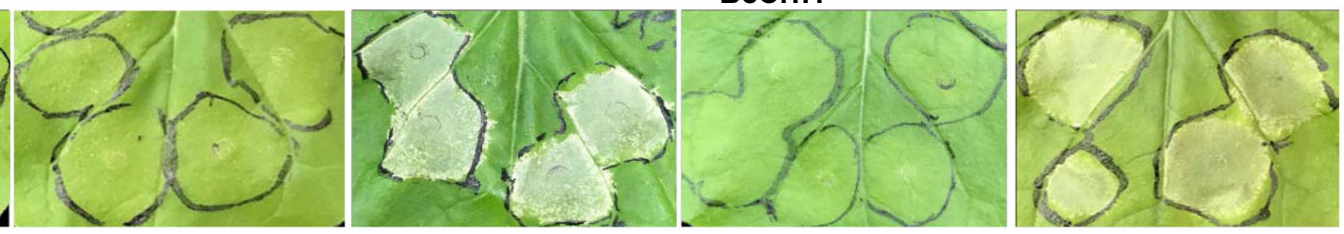

C

BcCrh175-144

SPPR3-BcCrh175-144

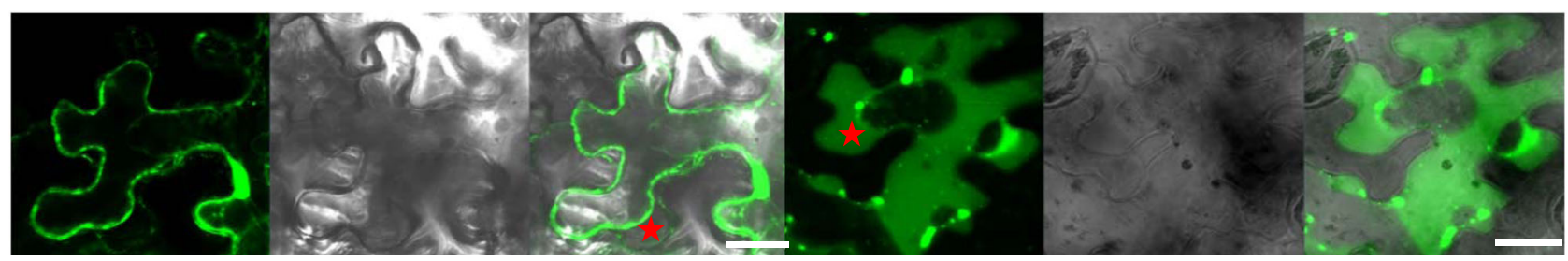

Fig. 2 A stretch of $\mathbf{3 5}$ amino acids of BcCrh1 is necessary and sufficient for the induction of plant cell death. a Schematic presentation of different $\mathrm{BcCrh1}$ fragments that were used in Agroinfiltration assays. Green-SP, Red-the minimal region that was found to be sufficient for induction of cell death, Blue-other regions of the tested fragment. b-c representative images of leaves following infiltrations with Agrobacterium strains that contain constructs encoding the different $\mathrm{BcCrh1}$ fragments. b Pictures were taken five days after Agroinfiltration; c Leaves were harvested two days after Agroinfiltration, submerged for $20 \mathrm{~min}$ in $0.8 \mathrm{M}$ mannitol to induce plasmolysis, and then samples were scanned by a confocal microscope. Typical apoplastic space between the cell wall and plasma membrane in plasmolysed plant cells is marked with red asterisks. Bars $=20 \mu \mathrm{m}$. All the above experiments were repeated at least three times with similar results. 
a

\begin{tabular}{|l|l|l|}
\hline MBcXYG1 \\
\hline SPcCrh1 \\
\hline SPcCrh1 & BcCrh1 ${ }^{\text {21-74 }}$ & MBcXYG1 \\
\hline
\end{tabular}
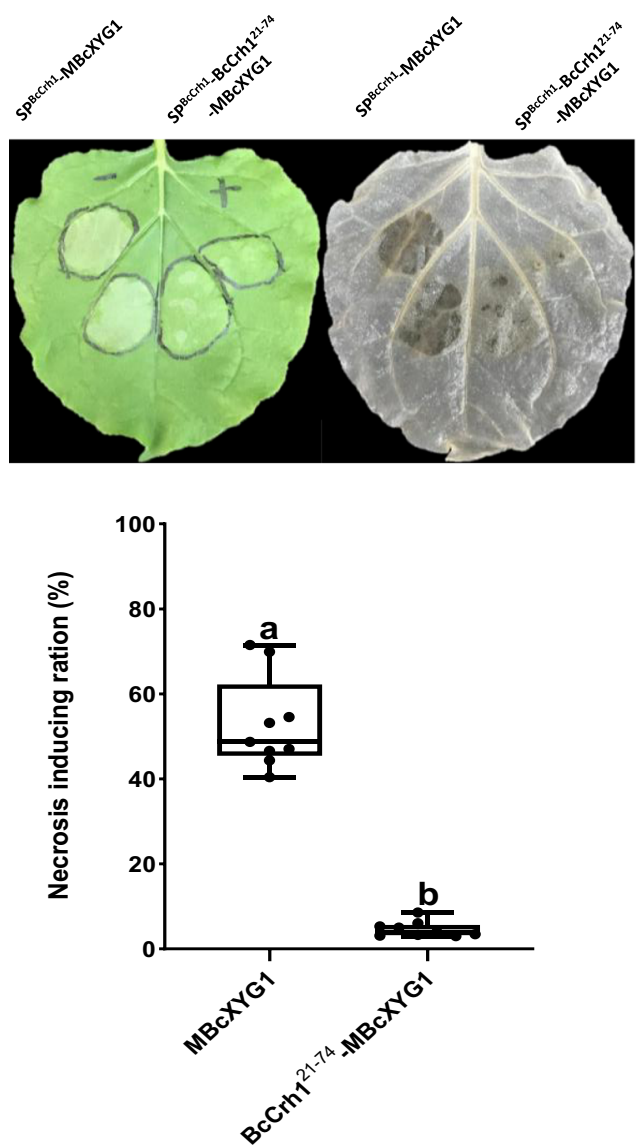

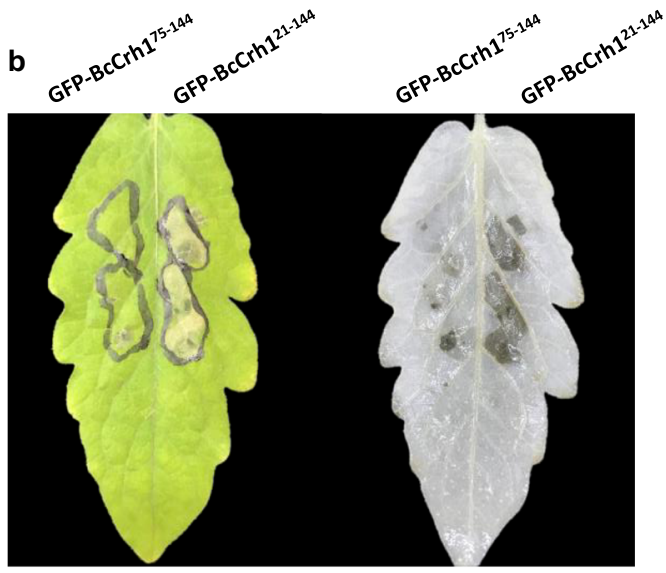

C

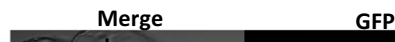

GFP

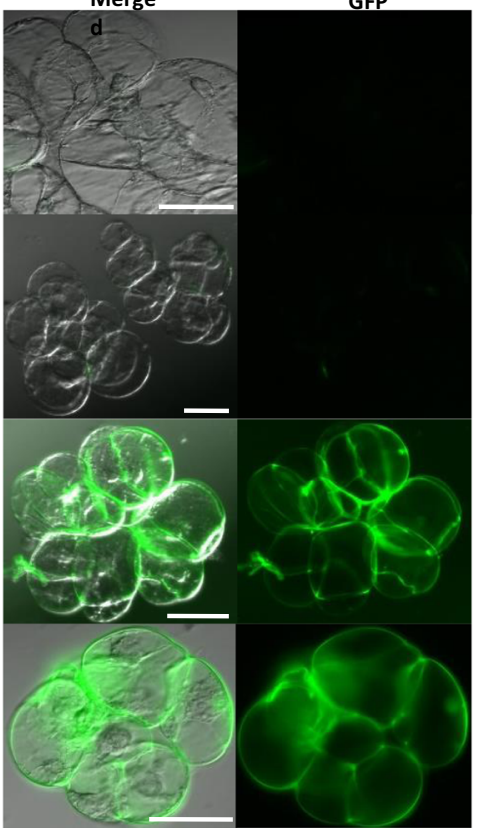

Fig. 3 A stretch of $\mathbf{5 3}$ amino acids at the $\mathbf{N}^{\prime}$ end of the protein mediates uptake of BcCrh1 by plant cells. Following analysis of a series of deletion constructs, a minimal region suspected of being necessary for protein uptake was defined. a To test the delivery of heterologous proteins using the putative

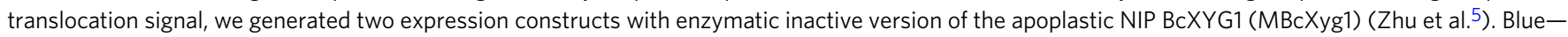
BcCrh1 SP, Red-MBcXyg1, Green-the suspected delivery sequence. Middle left- images of N. benthamiana leaves three days after Agroinfiltration. Leaves were bleached with ethanol (right images) and the necrotic area was calculated. Center lines of the boxplots show the medians, box limits indicate the 25th and 75th percentiles; whiskers cover the full range of values; all data points are plotted as black dots. Data are from 10 sample points from three

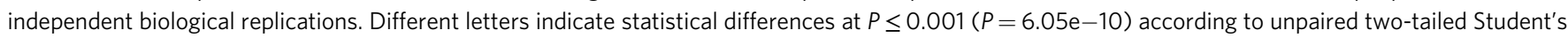
t-test. $\mathbf{b}$ Images of tomato leaves $48 \mathrm{~h}$ after infiltration with $11 \mu \mathrm{M}$ of purified $\mathrm{BcCrh1-derived} \mathrm{peptides,} \mathrm{BcCrh175-144}$ and BcCrh121-144. c Tomato cell cultures (MsK8) were incubated for $18 \mathrm{~h}$ with $5.5 \mu \mathrm{M}$ solution of GFP-tagged peptides (GFP-BcCrh175-144, GFP-BcCrh121-144, and GFP-BcCrh121-74), washed three times and visualized by a confocal microscope. Bars $=50 \mu \mathrm{m}$. The experiments were repeated three times with similar results.

leaves with purified BcCrh1 $1^{21-144}$ peptide caused significant cell death at $2 \mathrm{dpi}$, while treatment of leaves with the BcCrh1 ${ }^{75-144}$ peptide that lacks the putative uptake signal caused no visible symptoms (Fig. 3b). To obtain more direct evidence for the translocation function of the 53 aa region, we generated GFP fusion with the $\mathrm{BcCrh} 1^{21-144}, \mathrm{BcCrh} 1^{75-144}$ and $\mathrm{BcCrh} 1^{21-74}$ peptides and administered the purified proteins to a tomato cell suspension-culture. After $18 \mathrm{~h}$ of incubation, a GFP signal was detected in the cytoplasmic space of tomato cells incubated with the GFP-BcCrh1 $1^{21-144}$ and GFP-BcCrh1 $1^{21-74}$ protein, but not in cells incubated with the GFP-BcCrh1 $1^{75-144}$ protein (Fig. 3c). Taken together, these results confirmed that BcCrh1 is an intracellular, cell death-inducing effector and that translocation into the plant cells is mediated by the $\mathrm{N}^{\prime} 53$ amino acids residues of the protein.
BcCrh1 triggers PTI and induces host resistance against B. cinerea. Infiltration of $N$. benthamiana with Agrobacterium or purified protein solutions resulted in the accumulation of reactive oxygen species (ROS) and callose deposition (Fig. 4a, b). We also found that tomato defense-related marker genes were activated in response to infiltration with purified protein solutions (Fig. 4c). When leaves were inoculated with $B$. cinerea $48 \mathrm{~h}$ after infiltration with the protein, the infection was significantly reduced compared to control leaves pre-treated with GFP protein (Fig. 4d). Thus, along with cell death-inducing activity, BcCrh1 also triggers plant defense responses, similar to the vast majority of $B$. cinerea $\mathrm{NIPs}^{5,12-16,24,25}$. Since BcCrh1 does not induce cell death in $A$. thaliana (Supplementary Fig. 4b), we produced A. thaliana transgenic lines that express the BcCrh1 protein and tested their sensitivity to Botrytis. The BcCrh1-expressing plants were 
a

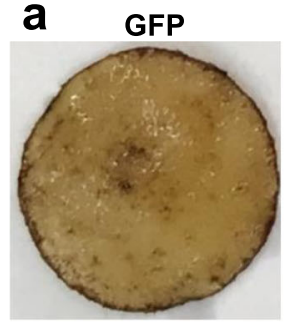

b
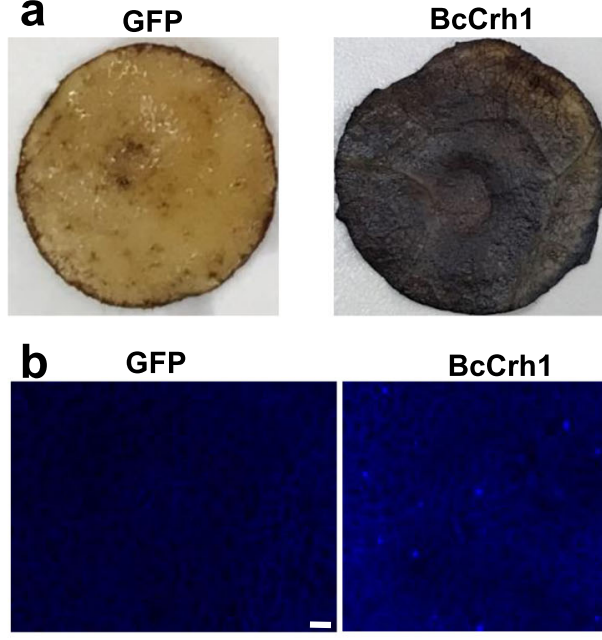

BcCrh1
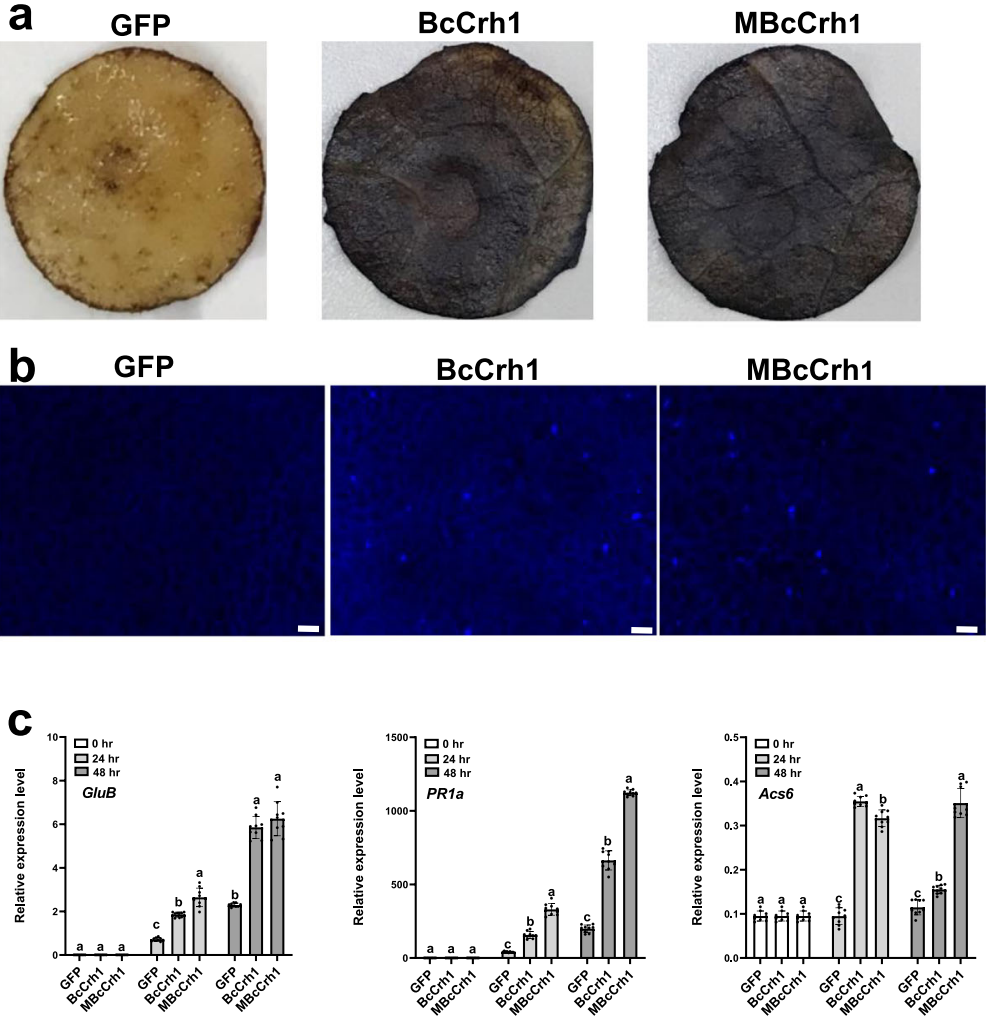

MBcCrh1
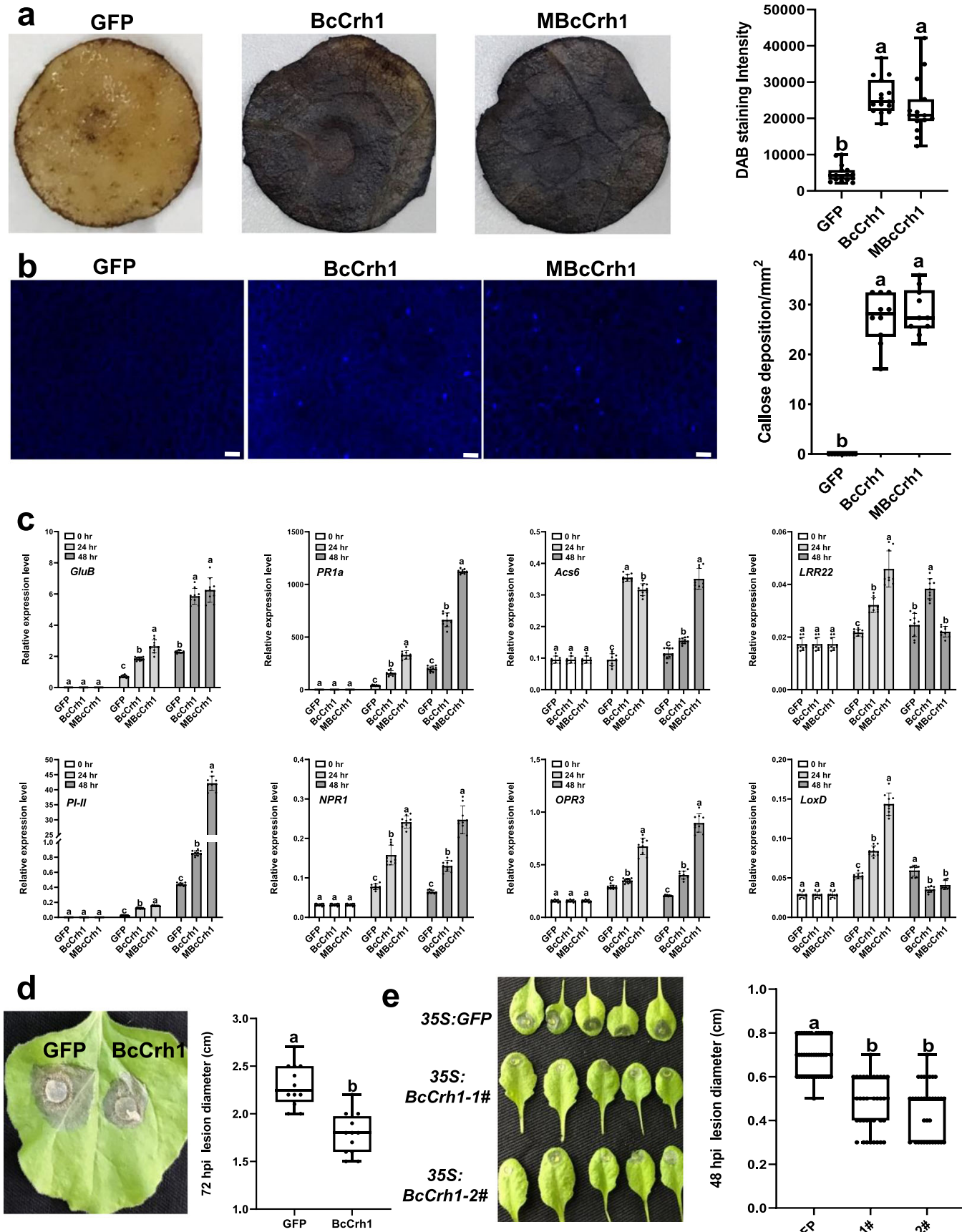

e

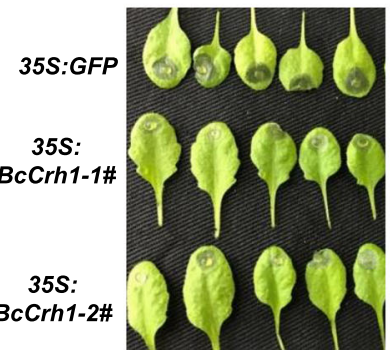

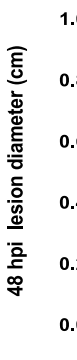

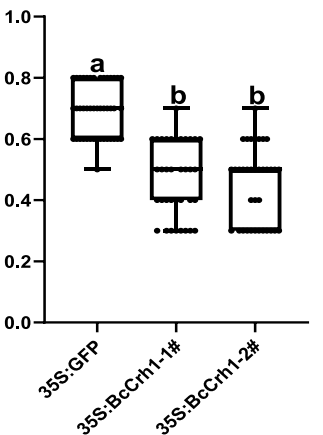

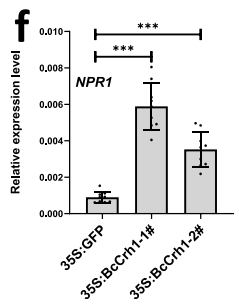
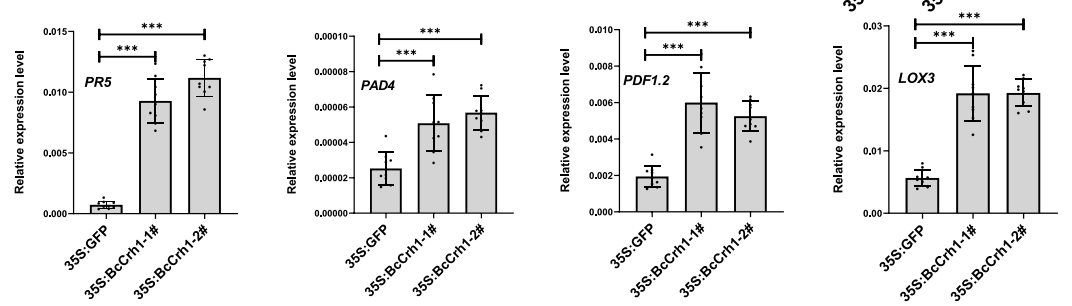

significantly less sensitive to infection by $B$. cinerea than control plants that were transformed with an empty vector, as determined by lesion size 3 dpi (Fig. 4e). Gene expression analysis showed induction of defense marker genes in the $\mathrm{BcCrh1}$-transgenic plants (Fig. 4f), which confirmed that reduced infection was due to induced plants defense.
BcCrh1 is expressed in planta and secreted from infection cushions. Crh proteins are fungal cell wall transglycosylases ${ }^{18-20}$. Nevertheless, BcCrh1 was found in the fungal secretome and is internalized by the plant cells, where it triggers plant cell death and defense responses, implying that it also affects fungal-plant interaction. To gain insights into the possible role of BcCrh1 in 
Fig. 4 BcCrh1 triggers plant immunity responses and enhances plant resistance to Botrytis infection. a ROS accumulation. N. benthamiana leaves were Agroinfiltrated with free GFP, and native or enzymatic inactive (MBcCrh1) BcCrh1. After $48 \mathrm{~h}$ samples were stained with DAB, they were decolorized with ethanol and staining intensity was quantified with ImageJ. b Calose deposition. $N$. benthamiana leaves were infiltrated with $11 \mu \mathrm{M}$ of purified proteins. Photomicrographs indicating callose deposition induced by BcCrh1 and MBcCrh1. After $24 \mathrm{~h}$ leaves were stained with aniline blue, bleached with ethanol, and images were captured with a fluorescent microscope $(B a r=50 \mu \mathrm{m})$. Callose levels were estimated by quantification of the number of spots per square millimeter using ImageJ. All data $(n=15$ in (a), $n=12$ in (b)) from three independent biological replicates are plotted as black dots. c Defense gene expression. Tomato leaves were infiltrated with purified proteins and relative expression levels of selected defense genes were determined by qRT-PCR 24 after 24 and $48 \mathrm{~h}$. Values represent mean \pm SD $(n=9)$ from three independent biological replicates and three technical replicates. $\mathbf{d}$ Infection assay. $N$. benthamiana leaves were infiltrated with $11 \mu \mathrm{M}$ of GFP (mock) or purified proteins, $48 \mathrm{~h}$ later the leaves were inoculated with $B$. cinerea mycelia plugs, the plants were incubated for an additional $72 \mathrm{~h}$ in a moist chamber and then symptoms were recorded. Data are from three independent experiments, each with four replications. e Infection assay of $A$. thaliana transgenic plants. Leaves of transgenic plants that express GFP (35S:GFP) or BcCrh1 (two independent plants, 35S:BcCrh-1\# and 35S:BcCrh-2\#) were inoculated with droplets of $B$. cinerea spore suspension, the plants were incubated in a moist chamber and symptoms were recorded $48 \mathrm{hpi}$. Images show all the inoculated leaves from a single plant. Graph shows data of three independent biological replications $(n=44,36,36)$. Whiskers of the boxplots in (a), (b), (d), and (e) show the minimum and maximum values; center lines of boxplots display the median values; box limits indicate the 25th and 75th percentiles. $\mathbf{f}$ Defense gene expression of transgenic $A$. thaliana plants. Relative expression levels of defenserelated marker genes were measured by qRT-PCR. Values are means $\pm S D(n=9)$ from three independent biological replicates and three technical replicates. Asterisks indicate significant difference between control (35S:GFP) and BcCrh1 transgenic lines according to one-way ANOVA, $P \leq 0.001$. In all other graphs, different letters indicate statistical differences at $P \leq 0.01$ according to one-way ANOVA.

infection development, we studied bccrh1 gene expression and protein localization in planta. Transcript levels of bccrh1 increased sharply following infection and reached a peak $12 \mathrm{hpi}$, in contrast to a much more gradual increase on solid Gamborg's $\mathrm{B} 5$ medium that peaked at $60 \mathrm{~h}$ post germination (Supplementary Fig. 7). To determine protein localization, a transgenic $B$. cinerea strain was produced with a bccrh1-gfp fusion gene under the control of the native $b c c r h 1$ promoter. During saprophytic growth on a culture medium, the protein was observed in the fungal vacuoles and ER, and accumulated to high levels in infection cushions (Fig. 5a, b). Infection assay of onion epidermis cells showed that the protein was secreted from hyphal tip 12-21 hpi, and from infection cushions at 36 hpi (Fig. 5c). Further evidence for BcCrh1 secretion was confirmed by Z-series projection, demonstrating that the GFP-BcCrh1 fusion protein is first delivered to the hyphal tip and later concentrates in infection cushions (Supplementary Fig. 8). Following secretion of the protein from the fungal cells, the GFP signal diffused in the surrounding apoplastic space and then accumulated inside the onion cells, including neighboring cells in regions of the tissue adjacent to the invasion area (Fig. 5d). These results show different expression and localization patterns of BcCrh1 during saprophytic and pathogenic development; During saprophytic growth, the gene is continuously expressed at moderate levels, and the protein is localized inside the fungal cells, while in planta, the gene is highly and transiently expressed following the first contact of the fungus with the plant, and the protein accumulates to high levels in infection structures before being released to the plant apoplast.

BcCrh1 is dispensable for $B$. cinerea pathogenicity and development. Deletion or overexpression of the bccrh1 gene had no visible effect on fungal development or pathogenicity (Fig. 6; Supplementary Fig. 9a-d). To determine whether the enzymatic activity activates a defense response that counteracts the cell death-inducing activity, two additional strains were generated that overexpress the inactive enzymatic protein (MBcCrh1) in wild-type (OE-MBcCrh1) and bccrh1 deletion $(\Delta / \mathrm{OE}-\mathrm{MBcCrh} 1)$ genetic background. Surprisingly, both strains were less virulent than the wild-type strain. In particular, the pathogenicity of the double mutant was severely reduced and symptoms were restricted to local lesions (Fig. 6a, b). Microscopic observation of infected leaves showed the $\Delta / \mathrm{OE}-\mathrm{MBcCrh} 1$ strain had a weaker penetration ability (Fig. 6c, d), which was associated with impaired infection cushion formation of this mutant compared with other strains (Supplementary Fig. 9d). Furthermore, pathogenicity of the $\Delta / \mathrm{OE}-\mathrm{MBcCrh} 1$ strain was at least partially restored by mechanical injury of leaves prior to infection (Supplementary Fig. 9e), suggesting a specific defect in penetration. The $\Delta / \mathrm{OE}-\mathrm{MBcCrh} 1$ strain also had developmental defects, including reduced sporulation and mycelium production, and an abnormal spore shape (Fig. 6e, f, Fig. 7c, d). However, there was no change in hyphal growth rate and spore germination (Supplementary Fig. 9a, b). These phenotypic changes were all more severe when the bccrh1 gene was deleted, indicating that they were caused by the accumulation of high levels of the MBcCrh1 protein. To test this hypothesis, we generated a strain that expresses the $\mathrm{MBcCrh} 1$ protein under control of the native $b c c r h 1$ promoter in the background of $\Delta b c c r h 1$ (strain $\Delta / \mathrm{NP}-\mathrm{MBcC}$ rh1). This strain did not show any developmental defects and it formed normal infection cushions and disease symptoms (Fig. 7), confirming that the developmental defects of the $\Delta / \mathrm{OE}$ MBcCrh1 strain resulted from high level accumulation of the enzyme inactive protein. The results also showed that the pathogenicity defects were caused at least in part by defects in plant invasion due to deformed infection cushions. Based on these findings, we predicted that BcCrh1 forms protein dimers necessary for the transglycosylase activity.

BcCrh1 forms homodimers as well as heterodimers with other BcCrh protein members. A yeast two-hybrid assay showed that both the native and the inactive BcCrh1 enzyme form protein dimers (Fig. 8a). The dimerization of BcCrh1 was further confirmed in vitro by a pull-down assay, in planta by Coimmunoprecipitation (Co-IP) and in vivo by BiFC (Fig. 8b-d). Sequence alignment of fungal Crh proteins revealed two conserved cysteine residues at positions 26 and 33 (Supplementary Fig. 1c), which form intramolecular disulfide bonds that might be important for proper folding and stability of the protein (Supplementary Fig. 2). Site-directed mutagenesis of either or both $C^{26}$ and $C^{33}$, as well as deletion of the sequence including $C^{26-33}$, all showed that $\mathrm{C}^{33}$, but not $\mathrm{C}^{26}$, was required for BcCrh1 homo-dimer formation (Fig. 8e). Despite the importance of $\mathrm{C}^{33}$ for homodimer formation and protein structure, the $\mathrm{BcCrh} 1^{\mathrm{C} 26 \mathrm{AC} 33 \mathrm{~A}}$ mutant protein retained full necrosis-inducing activity (Supplementary Fig. 3b, Supplementary Fig. 5), implying that the monomeric peptide was sufficient for induction of cell death, independent of the protein tertiary structure. However, the BcCrh1 $1^{\mathrm{C} 26 \mathrm{AC} 33 \mathrm{~A}}$ did not 

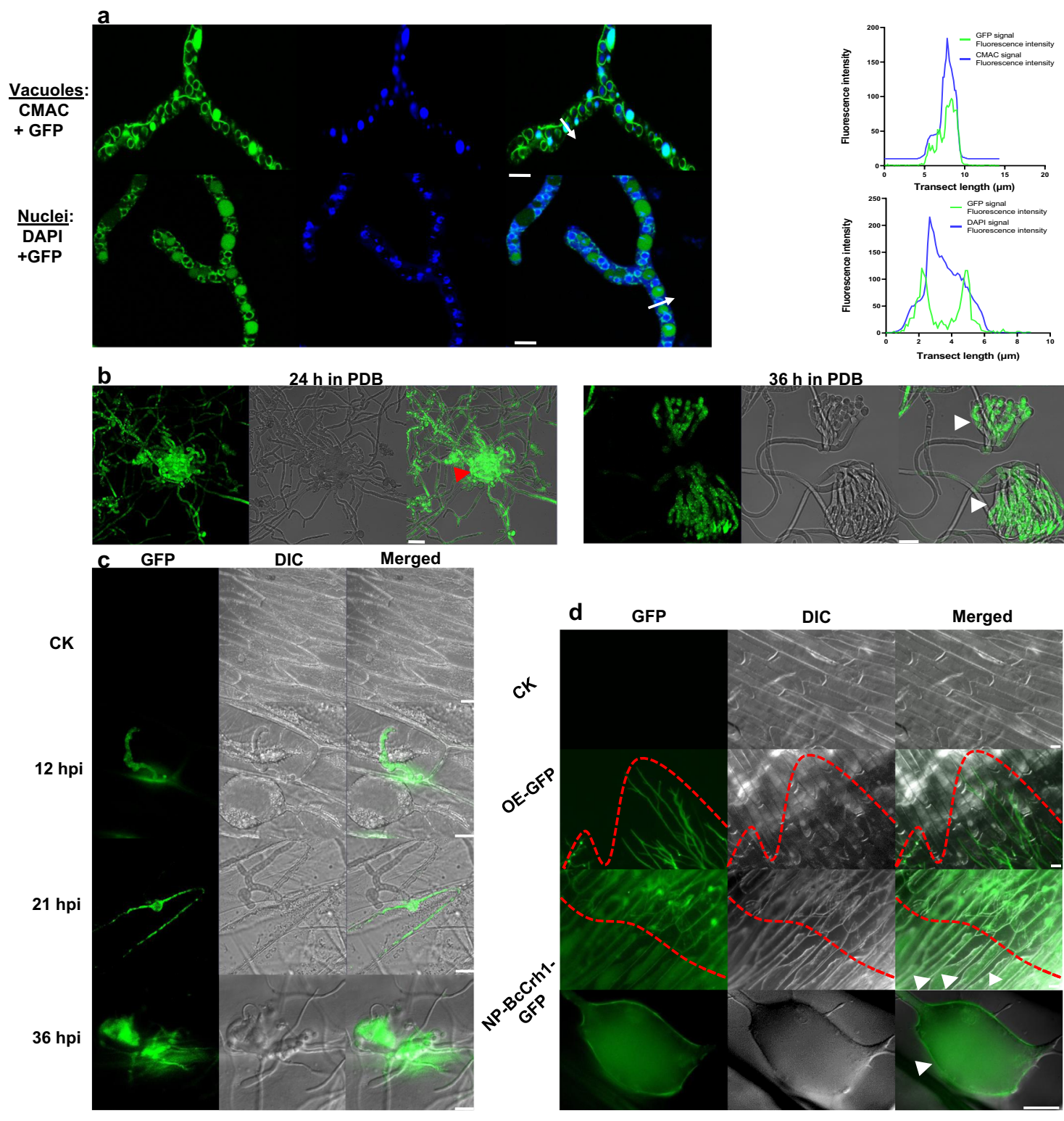

$24 \mathrm{~h}$ in PDB

$36 \mathrm{~h}$ in PDB
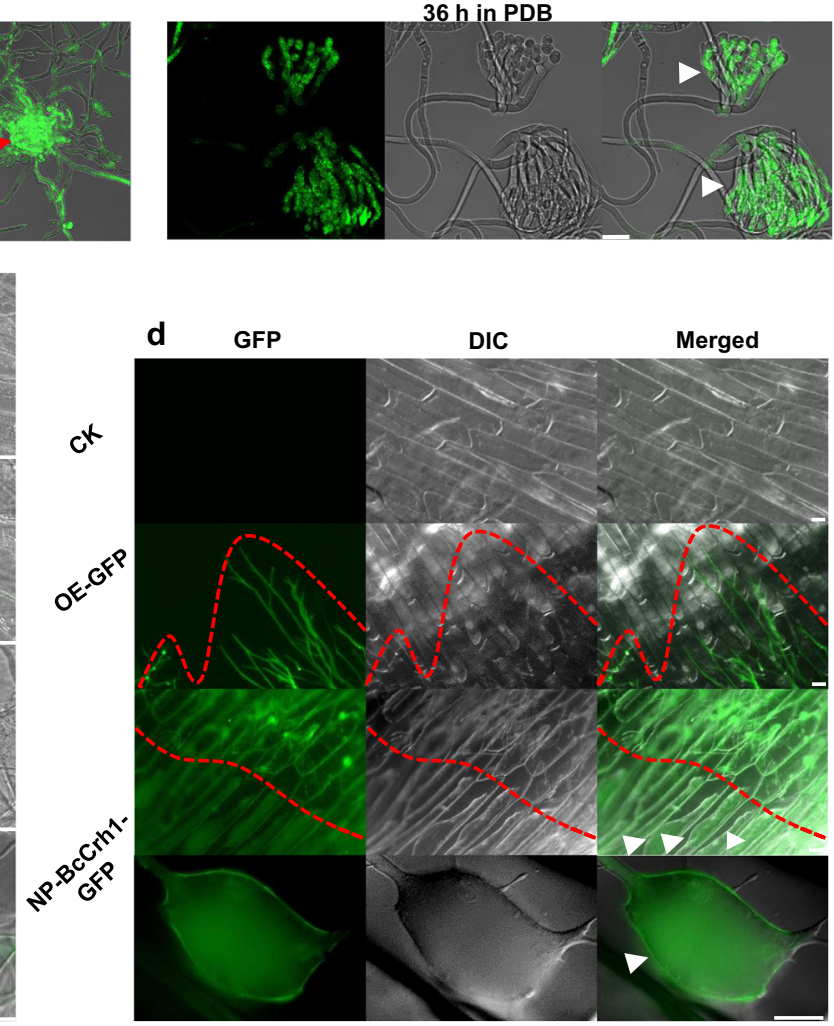

Fig. 5 Subcellular localization of BcCrh1 during saprophytic growth and host infection. a Intracellular localization of BcCrh1 during saprophytic growth. Spores of BcCrh1-GFP strain were cultured in liquid PDB medium for $12 \mathrm{~h}$, vacuoles (top) and nuclei (bottom) were stained with CMAC and DAPI, respectively, and samples were visualized using a Confocal microscope. Scale bars $=5 \mu \mathrm{m}$. The graph shows fluorescent intensity profiles of GFP/CMAC signals (top) and GFP/DAPI signals (bottom) in transects (white arrowheads). Y axis, GFP and CMAC or DAPI fluorescence intensity; $X$ axis, transect length $(\mu \mathrm{m})$. b Differential distribution of BcCrh1 in mycelia and infection cushions in vitro. Spores were germinated on a glass slide in PDB medium. At $24 \mathrm{~h}$ the GFP signal accumulates in the entire mycelium and in initiating infection cushions (left, marked with red arrow), at $36 \mathrm{~h}$ the signal is detected only in the mature infection cushions (right, indicated with white arrows). Scale bar $=20 \mu \mathrm{m}$. c-d Onion epidermis infection assay with cytoplasmic GFP and BcCrh1-GFP strains. c Images showing secretion of the protein from hyphal tips at early time points $(12,21 \mathrm{hpi})$ and from infection cushions at $36 \mathrm{hpi}$. Scale bars $=50 \mu \mathrm{m}$ at $12 \mathrm{hpi}$ and $20 \mu \mathrm{m}$ in all other images. $\mathbf{d}$ Intracellular localization of secreted BcCrh1-GFP protein in plasmolysed onion cells $45 \mathrm{hpi}$. Infection area is marked by red dashed line. BcCrh1-derived GFP signal is observed in the cytoplasmic space of plasmolysed onion cells outside the invasion area (marked with arrows). Scale bars $=20 \mu \mathrm{m}$. All the experiments were repeated three times with similar results.

complement the Congo red hypersensitivity of the yeast $\operatorname{crh} 1 \Delta \operatorname{crh} 2 \Delta$ double mutant, supporting the notion that protein dimerization may be necessary for the transglycosylation activity (Supplementary Fig. 6). Yeast two-hybrid assays with the three other $\mathrm{BcCrh}$ members showed that $\mathrm{BcCrh} 2$ and $\mathrm{BcCrh} 3$, but not $\mathrm{BcCrh} 4$, can also form homodimers, and that $\mathrm{BcCrh} 1, \mathrm{BcCrh} 2$ and $\mathrm{BcCrh} 3 \mathrm{can}$ form heterodimers with each other, all of which were independent of the enzymatic activity and mediated by the conserved cysteine residues (Fig. 8e, f). To verify our hypothesis that the excessive $\mathrm{MBcCrh} 1$ disrupts the function of the native $B$. cinerea $\mathrm{Crh}$ proteins by formation of enzymatic inactive protein dimers, we overexpressed the MBcCrh1 ${ }^{\mathrm{C} 26 \mathrm{AC} 33 \mathrm{~A}}$, which is unable to form dimers (Fig. $8 \mathrm{~d}$, e), in a $\Delta b c c r h 1$ background. In accordance with our prediction, this strain had no growth defects, and it caused normal disease symptoms (Fig. 7).

\section{Discussion}

$\mathrm{GH}$ are the largest GO group in B. cinerea secretome. The majority of the GHs are hydrolases of plant cell wall sugar polymers, such as cellulose, pectin, hemicellulose and other plant cell wall-associated polysaccharides ${ }^{26}$. Among them, a small number of proteins (catalytic NIPs) can also trigger cell death in 

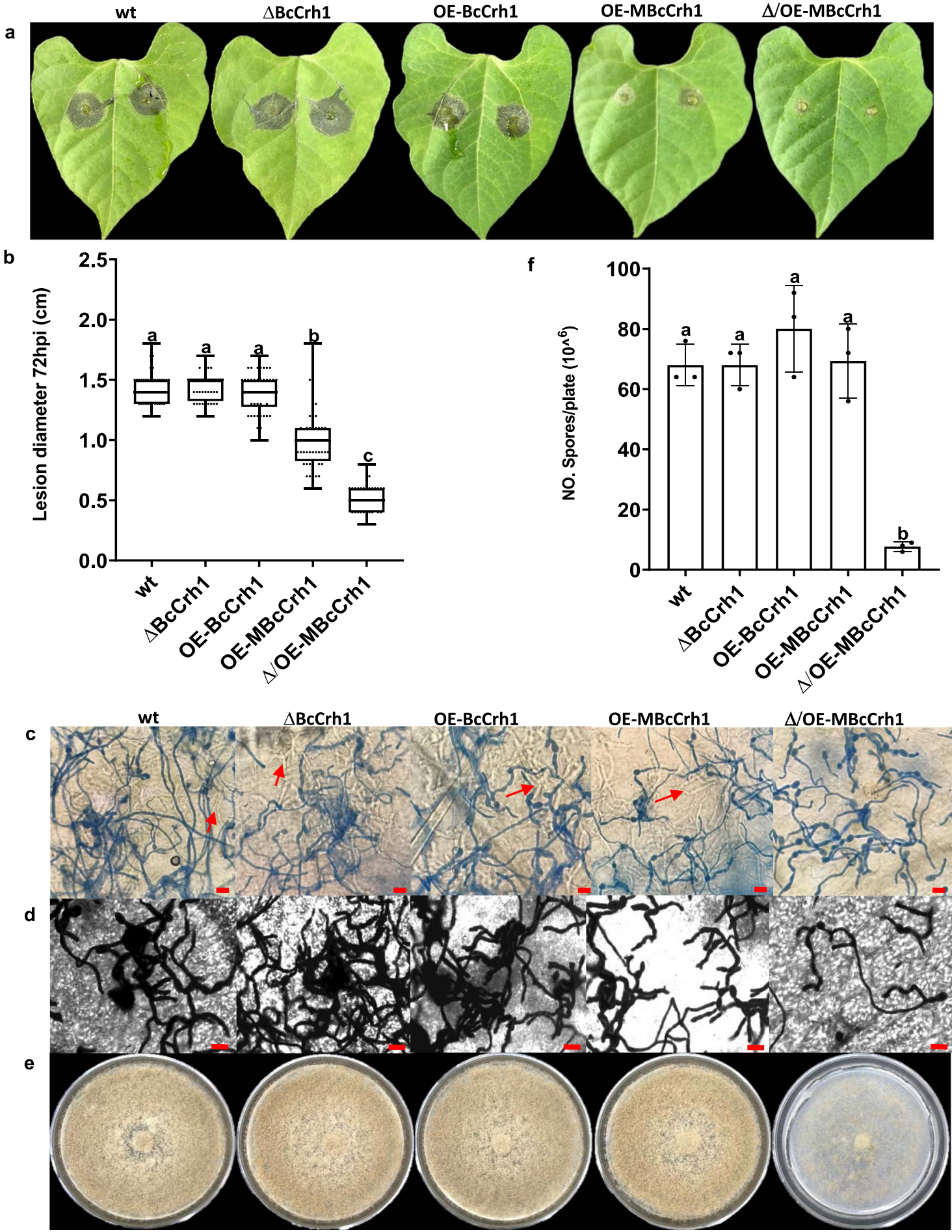

Fig. 6 Over expression of $\mathbf{M B c C} \mathbf{C r} 1$ causes reduced pathogenicity and developmental defects. $\mathbf{a}-\mathbf{b}$ Infection assay. Bean leaves were inoculated with spore suspensions of $B$. cinerea wild type (wt), bccrh1 deletion ( $\Delta \mathrm{BcC} C \mathrm{rh} 1)$, bccrh1 overexpression (OE-BcCrh1), and strains over-expressing the enzyme inactive (MBcCrh1) protein in wild type (OE-MBcCrh1) or bccrh1 deletion ( $\Delta / \mathrm{OE}-\mathrm{MBcCrh1)}$ genetic background. Symptoms were photographed and the lesion diameter was recorded $72 \mathrm{hpi}$. Box limits show the 25th and 75th percentiles. The center lines of boxplots indicate the medians values; whiskers extend to minimum and maximum values from the 25th and 75th percentiles; all data are indicated as black dots. At least 40 sample points from three independent biological replicates were used for statistical analysis. c-d Lactophenol cotton blue and lactophenol trypan blue staining of infected leaves. Leaf tissue was harvested at the designated time points and stained with lactophenol cotton blue, which stains only the exposed hyphae (c), and with lactophenol trypan blue, which stains both exposed and intracellular (red arrows) hyphae (d). Bar $=20 \mu \mathrm{m}$. Note the near absence of intracellular hyphae in the $\Delta / \mathrm{OE}-\mathrm{MBcCrh1}$ mutant, which indicates penetration defects. All the experiments were repeated three times with similar results. e-f $\mathrm{Mycelium}$ and spore production. Fungi were cultured on solid GB5-Glucose medium and grown at $20^{\circ} \mathrm{C}$ with continuous light. Pictures were taken (e) and spores counted after eight days of incubation. Data represent mean \pm SD from three independent biological replicates. Different letters in (b) and (f) indicate statistical differences at $P \leq 0.01$ according to one-way ANOVA. 
a

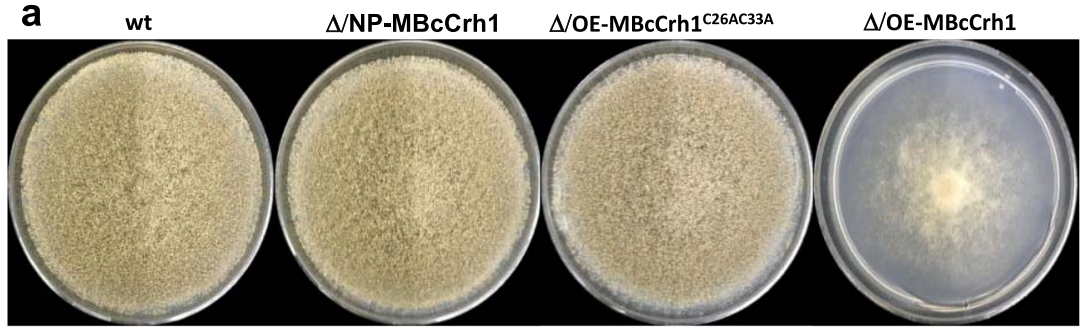

C

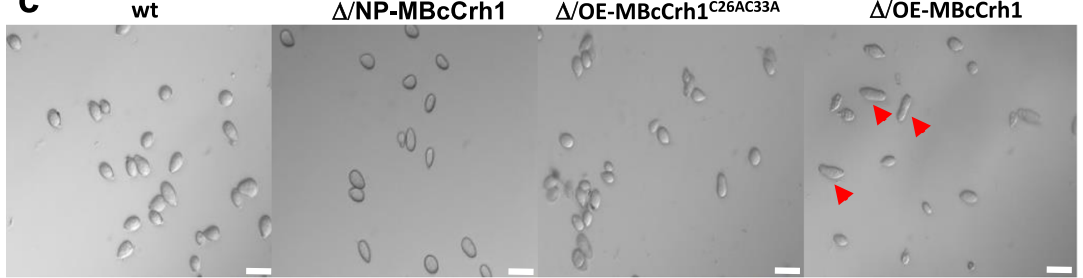

e

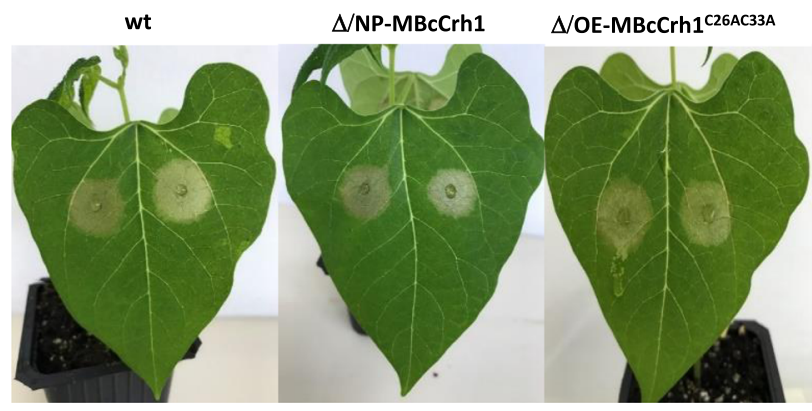

$\Delta$ OOE-MBcCrh1

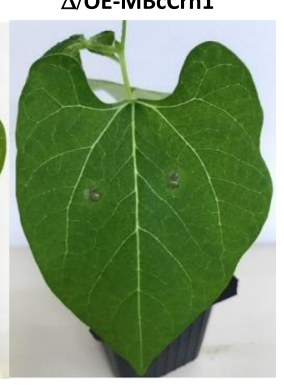

b

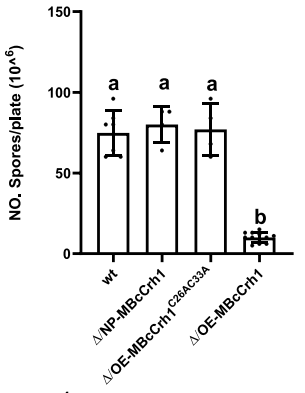

d

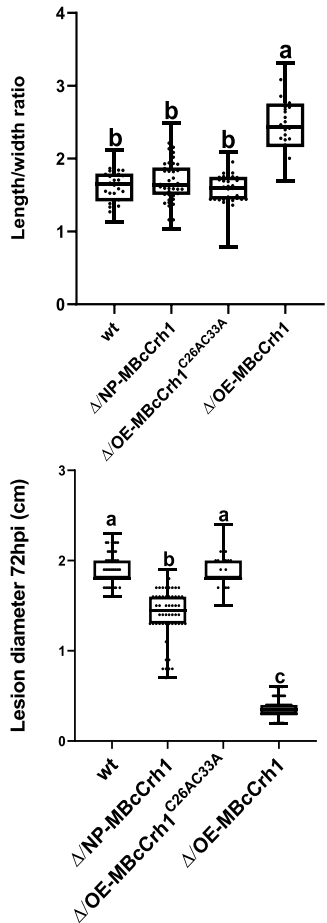

Fig. 7 The pathogenicity and developmental defects induced by $\mathbf{M B c C r h 1}$ depend on expression levels. The following strains were characterized: $B$. cinerea wild type (wt), enzyme inactive BcCrh1 with the native promoter in the background of bccrh1 deletion ( $\Delta / \mathrm{NP}-\mathrm{MBcC}$ rh1), overexpression of MBcCrh1 with mutation of $\mathrm{C} 26$ and $\mathrm{C} 33$ in a background of bccrh1 deletion ( $\triangle / \mathrm{OE}-\mathrm{MBcCrh1}{ }^{\mathrm{C} 26 \mathrm{AC} 33 \mathrm{~A}}$ ), and overexpression of MBcCrh1 in a background of bccrh1 deletion ( $\triangle / \mathrm{OE}-\mathrm{MBcC} \mathrm{rh1}$ ). a-d Spore production and shape. Fungi were cultured on solid GB5-Glucose medium and grown at $20^{\circ} \mathrm{C}$ with continuous light. Pictures of plates (a) and spores (c) were taken after eight days of incubation. Spores were harvested and average spore numbers (b) and spore dimensions (d) were determined. Arrows indicate spores of the $\Delta / \mathrm{OE}-\mathrm{MBcC}$ rh1 strain with abnormal morphology. For spore numbers, data represent mean \pm SD from three independent biological replications. For spore dimensions, the ratio of length/width was calculated. Data of at least 30 spores from three independent biological replications were used for statistical analysis. e-f Bean leaves were inoculated with spore suspensions of the different strains, pictures were taken and lesion size recorded $72 \mathrm{hpi}$. At least 32 sample data from three independent biological replications were used for statistical analysis. In boxplots ( $\mathbf{d}$ and $\mathbf{f}$ ), center lines represent the medians, box edges show the 25th and 75th percentiles; whiskers extend to minimum and maximum values from the 25th and 75th percentiles; all present data are indicated as black dots. Different letters in $\mathrm{d}$ and $\mathrm{f}$ indicate statistical differences at $P \leq 0.01$ according to one-way ANOVA.

plants $^{5,12-14}$, similar to the non-catalytic NIPs, such as NEP/ NELP $^{6,7}$ and ceratoplatanins ${ }^{25,27}$. Catalytic NIPs that have been characterized so far remain in the apoplast after secretion by the fungus, and their cell death-inducing activity is mediated by plant extracellular membrane components ${ }^{5,12,13}$. The $\mathrm{BcCrh} 1$ protein reported here represents a new class of NIPs: it has a defined catalytic domain but lacks a plant cell wall degrading activity, and its site of action is inside, rather than outside the plant cell. Furthermore, the cell death-inducing activity of $\mathrm{BcCrhl}$ is unexpected and surprising, since Crh family proteins catalyze crosslinking of sugar polymers in the fungal cell wall. Thus it lacks a clear connection to host-fungal interaction.

The Crh protein family is a conserved group of fungal-unique enzymes catalyzing cell wall maturation steps like chitin-glucan and chitin-chitin crosslinking ${ }^{18-20}$. Deletion of $S$. cerevisiae $C R H 1$ and $C R H 2$ genes affected cell wall integrity and proper development, particularly upon cell wall stress. Similar results were obtained in other yeast species ${ }^{18,19,28,29}$, while deleting the five $c r h$ gene members in A. fumigatus had almost no effect on fungal development ${ }^{20}$. Similarly, deletion of bccrhl did not affect B. cinerea development and only slightly increased sensitivity of the fungus to Congo red (Supplementary Fig. 9c). However, we found that the $\mathrm{BcCrh}$ proteins form dimers, and this dimerization seems to be necessary for the transglycosylase activity. Overproduction of the enzyme inactive monomer resulted in severe developmental defects, indicating that two intact monomers are necessary for proper function, and further supporting a redundant role of Crh proteins in filamentous species. These results reveal a new aspect in Crh proteins function, potentially leading to a better understanding of their mechanistic mode of action.

Under saprophytic conditions, the $\mathrm{BcCrh} 1$ protein was localized inside vacuoles and the ER. Still, during pathogenic 
a
SD-LW SD-LWHA+X- $\alpha$-gal

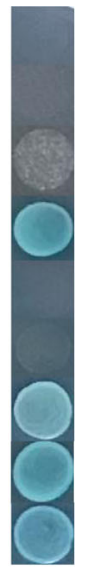

b

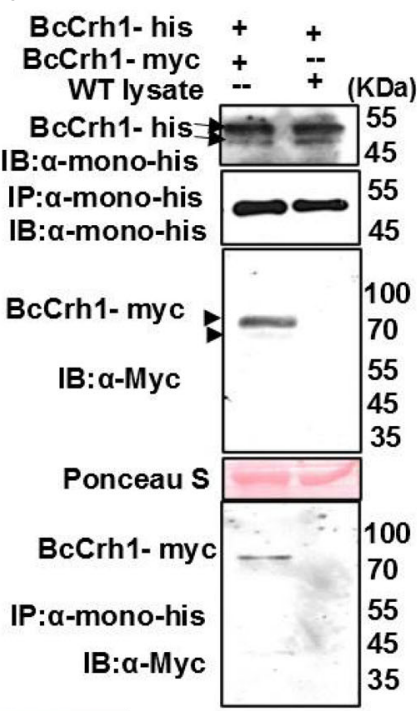

C

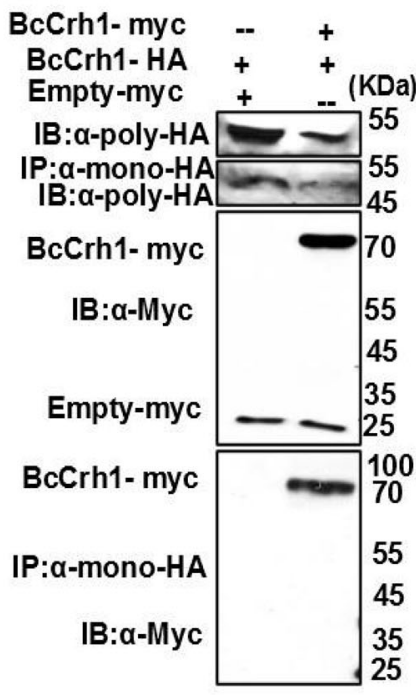

SD-LW SD-LWHA+X- $\alpha$-gal d

N'GFP

/C'GFP.

BcCrh1

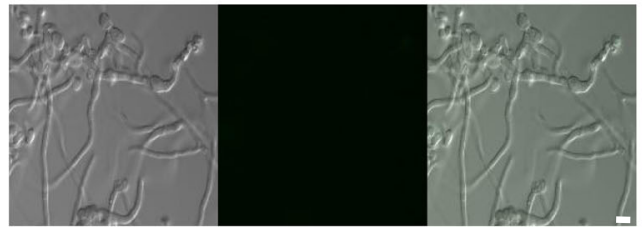

N'GFP-

BcCrh1

/C'GFP.

BcCrh1

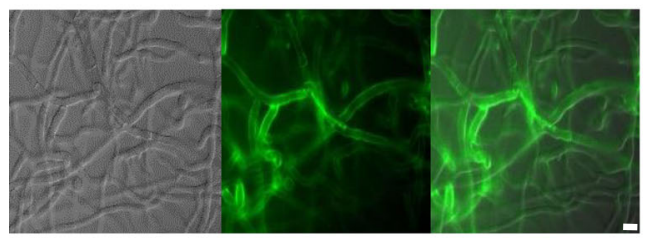

N'GFP-

BcCrh1 2 26AC33A

/C'GFP-

BcCrh1 126AC33A

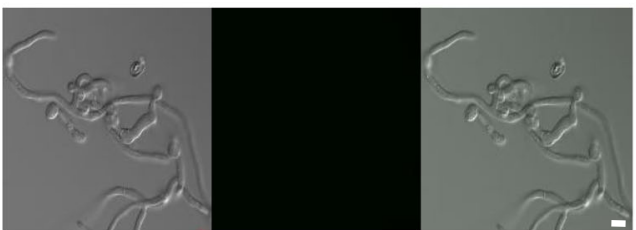

f e

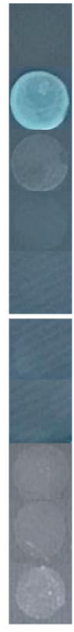

SD-LW SD-LWHA+X- $\alpha$-gal

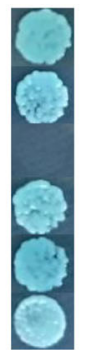

Fig. 8 BcCrh protein dimerization. a Yeast two-hybrid assay $(\mathrm{Y} 2 \mathrm{H})$ of $\mathrm{BcC}$ rh1 and MBcCrh1 homodimer formation. SD/-Trp-Leu medium was used to confirm the transformation events. SD/-Trp-Leu-His-Ade medium containing $\mathrm{X}$ - $\alpha$-gal was used to screen yeast strains with the positive protein-protein interaction. b-d Confirmation of BcCrh1 dimer formation by in vitro pull down assay (b), Co-immunoprecipitation (Co-IP) assay (c) and bimolecular fluorescence complementation (BiFC) assay (d). b For the in vitro pull-down assay, BcCrh1-myc was transiently expressed in N. benthamiana and pull down was performed with $\mathrm{BcC}$ Crh1-His recombinant protein conjugated with histidine beads. Protein from non-infiltrated leaves (WT lysate) was used as negative control. c For the Co-IP experiment, BcCrh1-myc or empty-myc were co-expressed with the BcCrh1-HA in N. benthamiana. BcCrh1-myc, but not empty-myc, co-immuno-precipitated with BcCrh1-HA conjugated to HA beads. c BiFC was conducted with B. cinerea transgenic strain expressing split GFP-BcCrh1 proteins (N'GFP-BcCrh1/C'GFP-BcCrh1). A strain expressing split GFP fused to BcCrh1 C26AC33A (N'GFP-BcCrh1 ${ }^{C 26 A C 33 A} / C^{\prime} G F P-B c C r h 1{ }^{C 26 A C 33 A}$ ) did not show any fluorescence, indicating a lack of dimer formation by this protein derivative. Bar $=10 \mu \mathrm{m}$. The experiments were repeated three times with similar results. e Effect of point mutations or deletion at the $\mathrm{N}^{\prime}$ part of $\mathrm{BcCrh1}$ on homo- and heterodimer formation. $\mathbf{f} \mathrm{Y} 2 \mathrm{H}$ assay of interaction between different BcCrh members. 
development, high levels of the protein were observed in developing infection cushions, before being secreted to the plant apoplast. The fungal strains in which $\mathrm{BcCrh}$ dimerization was blocked exhibited defective infection cushions and drastically reduced pathogenicity. This specific accumulation of BcCrh1 in infection cushions and aberrant infection cushions in the mutant suggests a possible role for $\mathrm{BcCrh} 1$ in infection cushion formation, which is associated with retardation of hyphal extension and hyper branching. It is possible that, following these pathogenicityspecific developmental events, the excessive enzyme, now serving as a virulent factor, is released from the mature infection cushions and induces plant cell death.

$\mathrm{BcCrh1}$ is a cytoplasmic effector, unlike previously isolated catalytic NIPs, which remain in the plant apoplast. Delivery of the protein to the plant nucleus (by addition of NLS) prevented cell death, indicating that the site of action is in the plant cytosol (Supplementary Fig. 3c). The uptake of $\mathrm{BcCrh} 1$ by plant cells is pathogen-independent and mediated by the first 53 aa of the mature $\mathrm{BcCrh} 1$ peptide. This feature places $\mathrm{BcCrh} 1$ in the category of cell-penetrating peptides (CPPs), a class of short peptides that facilitate cellular uptake of various molecules through endocytosis, macropinocytosis, and direct plasma membrane penetration ${ }^{30}$. Compared with animals, only a small number of CPPs have been reported in plants, including several that mediate effectors uptake, mainly from oomycetes. Specific motifs that have been associated with the delivery of oomycetes effectors include the RXLR motif common in Phytophthora effectors ${ }^{31-34}$, LXLFLAK in CRN proteins, and the CHCX-containing amino terminus motif in Albugo laibachii effector proteins ${ }^{35-37}$. RXLR effectors are assumed to be internalized via endocytosis in a pathogen-independent manner ${ }^{38,39}$, whereas the mechanisms of uptake of CRN and CHXC effectors remain elusive ${ }^{33}$. A relaxed RXLR-like motif has been proposed to be involved in the pathogen-independent, PI3P-mediated endocytosis of certain fungal effectors ${ }^{39-41}$. However to date, there is no known conserved motif that is shared by candidate cytoplasmic fungal effectors ${ }^{33,42}$. Similar to other fungal cytoplasmic effectors, the BcCrh1 translocation sequence does not contain any known conserved motifs and it lacks homology to sequences that mediate translocation of effectors in other fungi. Thus, the Nterminal uptake signal of $\mathrm{BcCrh} 1$ may be considered another class of CPP.

Similar to all other NIPs, along with plant cell death, BcCrh1 also elicits a PTI response. The cell death and PTI have opposite effects on disease development, which together with high redundancy of NIPs precludes a clear phenotype in deletion or over-expression fungal strains. However, $\mathrm{BcCrh} 1$ did not induce cell death in Arabidopsis, which enabled a better assessment of the induced PTI on disease development. Indeed, transgenic Arabidopsis plants that produce $\mathrm{BcCrh} 1$ were considerably less sensitive to $B$. cinerea, demonstrating the potential of BcCrh1, and possibly other NIPs, in engineering pathogen resistance in plants.

\footnotetext{
Methods

Fungi, bacteria, yeasts, and plants. Botrytis cinerea B05.10 and derived transgenic strains were routinely cultured on potato dextrose agar medium (PDA, Acumedia) at $22^{\circ} \mathrm{C}$ under continuous fluorescent light supplemented with near UV (black) light. The transgenic fungal strains were cultured on PDA amended with $100 \mu \mathrm{g} / \mathrm{ml}$ hygromycin B (Calbiochem) and/or $100 \mu \mathrm{g} / \mathrm{ml}$ Nourseothricin (Sigma-Aldrich). Escherichia coli strain DH5a and BL21 (DE3) were used for plasmid construction and propagation, and for protein expression, respectively. A. tumefaciens strain GV3101 was used for A. tumefaciens-mediated transient expression of proteins in plant leaves. All the bacteria were grown at $37^{\circ} \mathrm{C}$ on $\mathrm{LB}$ agar plates or in LB liquid medium supplemented with $100 \mu \mathrm{g} / \mathrm{ml}$ ampicillin and $50 \mu \mathrm{g} / \mathrm{ml}$ kanamycin. Yeast strain AH109 was used for yeast two-hybrid assay. For yeast complementation assays, S. cerevisiae BY4741 and GRA00743 strains were used. French bean (Phaseolus vulgaris L. genotype N9059), N. benthamiana, A. thaliana (ecotype Columbia-0), tomato (Solanum lycopersicum) cv. Hawaii
}

7998, and maize (Zea mays) cv. silver queen were grown in a growth room at $20^{\circ} \mathrm{C}$ (A. thaliana), or $25^{\circ} \mathrm{C}$ (all other plant species) with $16-\mathrm{h} / 8$-h light/dark cycle.

Bioinformatics analysis. The genomic sequence database of $B$. cinerea (https:// mycocosm.jgi.doe.gov/Botcil/Botcil.home.html) was used for Blast searches of $B$. cinerea genes. The SignalP 5.0 server (http://www.cbs.dtu.dk/services/SignalP/) was used to predict the presence of signal peptides and the location of their cleavage sites in the proteins ${ }^{44}$. TMHMM Server v. 2.0 (http://www.cbs.dtu.dk/services/

TMHMM/) was used for the prediction of transmembrane helices in proteins ${ }^{45}$. The conserved protein domain search was performed by SMART MODE (http://smart emblheidelberg.de/smart/change_mode.pl) ${ }^{46}$, while the databases NCBI and UniProt were used for BLASTp searches. Multiple Sequence Alignment (MSA) was performed by Clustal Omega (https://www.ebi.ac.uk/Tools/msa/) ${ }^{47}$ and MView Version 1.63 was used to present the result. HHpred (https://toolkit.tuebingen.mpg de/tools/hhpred $)^{48}$ was used for the prediction of the 3D structural models of the template from PDB database. The template with the highest sequence identity was used for modeling. To produce the pairwise alignment between the two proteins, ConSurf was used to collect homologues ${ }^{49}$. Sequences were collected from the clean UniProt database with 95\% maximal identity between sequences and minimal 35\% identity for homologues. Sequences that introduced large gaps into the alignment were discarded, and the pairwise alignment of BcCrh1 and 6IBW was deduced from the multiple sequence alignment. MODELLER (https://salilab.org/modeller/) was used to produce different models ${ }^{50}$, and each model underwent a short energy minimization using GROMACS and the AMBER99SB-ILDN force field (https:// www.nvidia.com/es-la/data-center/gpu-accelerated-applications/gromacs/) ${ }^{51,52}$. Finally, the model result with the predicted lowest energy was chosen. The models cover amino acids 25 to 275 (according to the amino acids numbering in UniProt entry A0A384J6C4). Each model underwent a short energy minimization using GROMACS and the AMBER99SB-ILDN force field. To test potential dimerization interfaces, PISA (https://www.ebi.ac.uk/pdbe/pisa/) ${ }^{53}$ and the template's crystal structure (6IBW) were used. PyMOL 2.4 (https://pymol.org/2/) for visualization of $3 \mathrm{D}$ structural models.

Construction of plasmid DNA. Primers used in this study are listed in Supplementary Table S1. All constructs were sequence-verified to confirm their integrity before further manipulation. Binary plasmids based on $2 \times 35$ S-MCS-eGFP (pCNG) were used for gene expression in plants ( $N$. benthamiana and $A$. thaliana $)^{54}$. The ORFs of target genes were amplified from cDNA with Phusion High-Fidelity DNA Polymerases (NEB). Signal peptide sequence deletion, Cterminus truncations, the addition of nuclear localization (NLS) or myristoylation (CBL) signals, and site-directed PCR mutagenesis were conducted on the target sequences according to specific requirements. The fragments were cloned into linearized pCNG (the vector plasmid was excised with XbaI and BamHI) and fused to the eGFP using an E. coli DH5a-mediated DNA assembly method ${ }^{55}$. For the generation of $B$. cinerea mutant strains, several plasmids were constructed. For deletion of the $b c c r h 1$ gene, $500 \mathrm{bp}$ each of $b c c r h 1-5^{\prime}$ and $b c c r h 1-3^{\prime}$ flanking regions were amplified from B05.10 genomic DNA and added on each side of a hygromycin-resistance cassette to produce the bccrh1 deletion plasmid pTZ$\Delta b c c r h 1$. To construct the $b c c r h 1$ over-expression vector, the full-length $b c c r h 1$ $\mathrm{ORF}$ fused to an HA tag at the C-terminus was cloned into the $\mathrm{pH} 2 \mathrm{G}$ vector ${ }^{5}$. To generate an enzyme inactive $\mathrm{BcCrh} 1$ protein, the catalytic residues were mutated (E120Q/D122H/E124Q) by site-directed PCR mutagenesis using template cDNA, and the amplification fragment was cloned into pJET plasmid to generate the pJET$\mathrm{MBcCrh} 1$ vector. For generation of fungal strains that overexpress the MBcCrh1 in a wild type and bccrh1 deletion background, the Mbccrh1 clone was introduced into pNAN-OGG (contains a nourseothricin resistance cassette) ${ }^{56}$, between the Aspergillus nidulans POliC promoter (for overexpression) or the bcchl promoter, and $B$. cinerea Tgluc terminator. Mutagenesis of the conserved cysteine residues at the $\mathrm{N}^{\prime}$ end (C26A/C33A/E120Q/D122H/E124Q) were generated by site-directed $\mathrm{PCR}$ mutagenesis and the amplified fragments were cloned into a pNAN-OGG vector. For the BiFC assay in B. cinerea, split-GFP construction (N'GFP-BcCrh1/C' GFP-BcCrh1 under the control of bidirectional H2B promoter) was generated. For protein expression and purification, the sequence encoding mature proteins without the signal peptide was cloned into pET-14b $(+)$ (Novagen) to generate the expression vector $\mathrm{pET} 14 \mathrm{~b}-6 \mathrm{xHis}-\mathrm{BcCrh} 1$. Expression vectors of truncated-BcCrh1 were constructed in a similar way. To generate the constructs used for a yeast-twohybrid assay $(\mathrm{Y} 2 \mathrm{H})$, the ORFs of target genes without the secretion sequence were amplified using PCR or site-directed mutagenesis PCR (C26A, C33A, C26A/C33A, and $\triangle 21-38)$, and the PCR amplification products were cloned into the pGADT7 and pGBKT7 yeast two-hybrid vectors. To generate the vectors used for yeast complementation assay, the ORFs of target genes were introduced into YEp352 under the control of $\mathrm{CRH} 2$ promoter and terminator ${ }^{43}$

DNA and RNA analyses. Genomic DNA and total RNA extractions were performed by Extract-N-Amp ${ }^{\text {tx }}$ Tissue PCR Kits (Sigma/aldrich) and TRIzol reagent (Sigma/Invitrogen). For cDNA synthesis, total RNA was treated with DNase I (Thermo Scientific) and then the first-strand cDNA was synthesized from $1 \mu \mathrm{g}$ of DNA-free RNA using the RevertAid First Strand cDNA Synthesis Kit (Thermo Scientific). qRT-PCR was performed with SYBR Premix Ex Taq II (Takara, Dalian, 
China) using Mx3000P (Stratagene, La Jolla, CA, USA) or StepOne (Applied Biosystems) Real-time PCR instruments. Relative fold change of mRNA levels was normalized with the B. cinerea bcgpdh (BC1G_05277), S. lycopersicum actin gene, or A. thaliana UBQ10 gene (AT4g05320).

A. tumefaciens-mediated transient expression. Constructs were introduced into A. tumefaciens strain GV3101 by electroporation, and Agrobacterium infiltration assays were performed on $N$. benthamiana plants that were grown for $4-5$ weeks in the greenhouse as described previously ${ }^{57}$. Briefly, A. tumefaciens strains were inoculated into $1 \mathrm{ml}$ of LB liquid medium containing $50 \mu \mathrm{g} / \mathrm{ml}$ of kanamycin and $50 \mu \mathrm{g} / \mathrm{ml}$ of rifampin. The bateria were incubated at $28^{\circ} \mathrm{C}$ overnight, the cells were harvested by centrifugation at $2000 \times g$ for $5 \mathrm{~min}$ and then resuspended in the infiltration buffer ( $10 \mathrm{~mm} \mathrm{MgCl} 2,10 \mathrm{~mm}$ MES, pH 5.6, $100 \mu \mathrm{M}$ acetosyringone) and diluted to $\mathrm{OD} 600=0.5-1$. Unless otherwise mentioned, leaves were photographed five days after infiltration. Expression of all the proteins was verified by western blot analysis 2-3 d after Agrobacterium infiltration (Supplementary Fig. 11).

Leaf infiltration assay with purified proteins. To test the cell death-inducing activity of recombinant proteins, $2.75-55 \mu \mathrm{M}$ protein solutions were infiltrated into leaves of $N$. benthamiana, and leaves of S. lycopersicum. Leaves of $A$. thaliana and $Z$. mays were infiltrated with $11 \mu \mathrm{M}$ protein solution. Infiltrated leaves were photographed 2-3 days after infiltration. To test the induced plant defense responses, total RNA was extracted from S. lycopersicum leaves at 0,24 , and $48 \mathrm{~h}$ after infiltration with recombinant protein solutions $(11 \mu \mathrm{M})$ and $\mathrm{QRT}-\mathrm{PCR}$ analysis was performed for measurement of plant defense-related gene expression levels. The leaves of $N$. benthamiana were infiltrated with $11 \mu \mathrm{M}$ purified proteins, then sampled at $24 \mathrm{~h}$ for quantification of callose deposition.

Uptake assay of GFP-labeled proteins by plant cells. Tomato cell cultures (MsK8) were incubated for $30 \mathrm{~min}$ with incubation buffer $(10 \mathrm{mM}$ phosphate buffer, $\mathrm{pH} 7$, and $0.1 \% \mathrm{BSA}$ ), followed by the addition of GFP-tagged peptide solutions $(5.5 \mu \mathrm{M})$ for $18 \mathrm{~h}$. The cells were subsequently washed three times in incubation buffer and visualized with confocal laser microscopy.

Protein analysis. Plasmids used for protein expression were transformed into E. coli strain BL21 (DE3). Expression and purification of the recombinant proteins were performed using Ni-NTA resin (GE Healthcare) according to previously described protocol ${ }^{58}$. In brief, $500 \mathrm{~mL}$ of LB medium (supplemented with $100 \mu \mathrm{g} / \mathrm{ml}$ ampicillin) was inoculated with $5 \mathrm{ml}$ of pre-cultured E. coli BL21 strain containing the desired plasmid and the cultures were incubated at $37^{\circ} \mathrm{C}$ with shaking until they reached $\mathrm{OD}_{600}=0.8$. The culture was cooled to $16^{\circ} \mathrm{C}$, IPTG (isopropyl- $\beta$-D-thiogalactoside) was added to a final concentration of $0.2 \mathrm{mM}$, and the cultures were incubated with shaking overnight. Cells were harvested by centrifugation at $5,000 \mathrm{~g}$ for $10 \mathrm{~min}$ and the pellet was resuspended in buffer A $(20 \mathrm{mM}$ sodium phosphate, $300 \mathrm{mM}$ sodium chloride (PBS) with $10 \mathrm{mM}$ imidazole; $\mathrm{pH}$ 7.4). The cells were busted by sonication, centrifuged at $23,000 \times g$ for $30 \mathrm{~min}$ at $4{ }^{\circ} \mathrm{C}$, and the supernatant was loaded onto a Ni-NTA column. The columns was washed with buffer B (PBS with $25 \mathrm{mM}$ imidazole; $\mathrm{pH}$ 7.4), and eluted with buffer $\mathrm{C}$ (PBS with $250 \mathrm{mM}$ imidazole; $\mathrm{pH}$ 7.4). The protein was further cleaned and concentrated by using a $10-\mathrm{kDa}$ molecular weight cut-off Amicon Ultra centrifugal filter (Millipore).

For analysis of proteins following Agroinfiltration, leaves were grounded in extraction buffer [ $20 \mathrm{mM}$ Tris- $\mathrm{HCl}$, pH 7.5, $100 \mathrm{mM} \mathrm{NaCl}, 1 \mathrm{mM}$ EDTA, $2 \mathrm{mM}$ NaF, $2 \mathrm{mM} \mathrm{Na}_{3} \mathrm{VO}_{4}, 1 \mathrm{mM}$ dithiothreitol (DTT), $0.5 \%$ Triton X-100, 10\% glycerol and $1 \times$ protease inhibitor cocktail] and the extract was centrifuged at $12,500 \times g$ for $20 \mathrm{~min}$ at $4{ }^{\circ} \mathrm{C}$. The cell lysate was harvested, boiled with $4 \times$ SDS protein loading buffer at $95^{\circ} \mathrm{C}$ for $15 \mathrm{~min}$, and detected by immunoblotting with indicated antibodies.

Fluorescence and confocal microscopy. Samples were collected from N. benthamiana leaves two days after Agroinfiltration. For plasmolysis, samples were infiltrated with $0.8 \mathrm{M}$ mannitol solution for $20 \mathrm{~min}$. For subcellular localization of GFP-labeled BcCrhl in fungal cells during saprophytic growth, conidia were suspended in PDB and $10 \mu$ droplets of spore suspension containing $1 \times 10^{4}$ conidia $\mathrm{ml}^{-1}$ were placed on a glass slide and incubated at $20^{\circ} \mathrm{C}$. Confocal microscopy was performed with a Zeiss LSM780 confocal microscope system and ZEISS ZEN 3.0 (blue edition) imaging Software ${ }^{59}$. Epifluorescence and light microscopy were performed with a Zeiss Axio imager M1 microscope and Carl Zeiss AxioVision Rel. 4.8 Software. Differential interference microscopy (DIC) was used for bright field images. DAPI filter (340-390 and 420-470 $\mathrm{nm}$ excitation and emission, respectively) was used for visualization of DAPI-stained nuclei and CMAC stained vacuoles. eGFP and mCherry fluorescence were collected using excitation laser wavelengths of 488 and $561 \mathrm{~nm}$, respectively. For GFP, emission was collected in the range of $493-535 \mathrm{~nm}$. Images were captured with a Zeiss AxioCam MRm camera.
Transformation of $B$. cinerea and characterization of the transformants. PEGmediated protoplast transformation of B. cinerea was performed according to published protocols ${ }^{60}$. Transgenic strains are listed in Supplementary Table S2. At least three independent single spore isolates from independent colonies were obtained for each strain. The $\triangle \mathrm{BcCrh} 1$ colonies were confirmed by PCR to verify deletion of the $b c c r h 1$ gene and the level of $b c c r h 1$ gene expression in overexpression strains was determined by qRT-PCR. The phenotypic assays, including mycelial growth rates, conidiation, conidial germination and stress tolerance, were performed according to a previous description ${ }^{60,61}$

Pathogenicity and infection-related assays. The pathogenicity assay with $B$ cinerea was performed as described previously $y^{60}$. For bean plants, the first two primary leaves of a 10-day-old plants were inoculated with $7.5 \mu \mathrm{l}$ of conidia suspension containing $2 \times 10^{5}$ conidia $\mathrm{ml}^{-1}$. For $A$. thaliana plants, leaves of $4-5$-week-old plants were similarly inoculated with $5 \mu \mathrm{l}$ conidial suspension. Infection intensity was evaluated by measurement of lesion diameter $72 \mathrm{hpi}$. For the infection cushion formation assay, conidia were suspended in GB5 $+2 \%$ Glucose, $10 \mu \mathrm{l}$ were placed on glass slides and incubated in a moistened chamber at $20^{\circ} \mathrm{C}$ for $36 \mathrm{~h}$. For localization of GFPlabeled $\mathrm{BcCrh} 1$ during infection, conidia were suspended in PDB medium and $10 \mu \mathrm{l}$ droplets of spore suspension containing $1 \times 10^{4}$ conidia ml $^{-1}$ were placed on onion epidermal cells and incubated at $20^{\circ} \mathrm{C}$ in moist conditions. Samples were prepared for microscopy observation at designated time points.

Staining dyes and procedures. Cotton blue and trypan blue staining of fungal hyphae for the penetration assay of plant leaves were performed according to published procedures ${ }^{62}$. DAB staining of ROS and aniline blue staining of callose depositions were conducted based on the published descriptions ${ }^{63,64}$. Briefly, detached plant leaves were soaked overnight in one of the following staining solutions: lactophenol cotton blue $(0.5 \mathrm{mg} / \mathrm{ml}$ of cotton blue dissolved in lactophenol containing an equal volume of lactic acid, glycerol, liquid phenol and $\left.\mathrm{ddH}_{2} \mathrm{O}\right)$, trypan blue $(2.5 \mathrm{mg} / \mathrm{ml}$ of trypan blue dissolved in lactophenol containing an equal volume of lactic acid, glycerol, liquid phenol and $\left.\mathrm{ddH}_{2} \mathrm{O}\right), \mathrm{DAB}(1 \mathrm{mg} / \mathrm{ml}$ of $\mathrm{DAB}$ dissolved in $\mathrm{ddH} 2 \mathrm{O}, \mathrm{pH} 3.6)$, or aniline blue $(10 \mathrm{mg} / \mathrm{ml}$ of aniline blue in $150 \mathrm{mM} \mathrm{K}_{2} \mathrm{HPO}_{4}, \mathrm{pH} 9.5$ ). Following incubation the leaves were transferred into distaining solution and incubated at room temperature with gentle shaking. Nuclei staining with DAPI was performed as previously described ${ }^{65}$. Briefly, hyphae were incubated for $10 \mathrm{~min}$ in the dark in PBS containing $1 \mu \mathrm{g} / \mathrm{ml}$ DAPI, the staining solution was removed and the sample was washed with PBS. For staining of vacuoles with CMAC, spores were germinated on cover slips, the cultures were incubated in GB medium with $10 \mu \mathrm{M}$ CMAC for 20 min and then washed with fresh GB medium.

Transformation of A. thaliana. Agrobacterium-mediated transformation of A. thaliana flowers was performed using the floral dip method ${ }^{66}$. Transgenic plants were selected on half-strength Murashige and Skoog medium with $0.5 \%$ sucrose, $0.8 \%$ agar and $2.5 \mathrm{mM}$ MES at $\mathrm{pH} 5.7$, containing $50 \mu \mathrm{g} / \mathrm{ml}$ of kanamycin. Three independent transgenic lines were generated with $b c c r h 1$ overexpression and empty vector (control) plasmids. Protein expression was confirmed by western blot analysis (Supplementary Fig. 11). Homozygous T3 seeds were selected and used for all experiments.

\section{Yeast transformation, yeast two-hybrid assay, and yeast complementation} assay. The Matchmaker Gold Yeast Two-Hybrid (Y2H) System (Clontech, Palo Alto, CA, USA) was used to verify proteins interaction. The bait (pGBKT7) and prey (pGADT7) plasmids were co-transformed into yeast strain AH109 using the LiAc/SS carrier DNA/PEG method ${ }^{67}$. Yeast transformants were screened on selective dropout (SD)/-Trp-Leu medium to select yeast cells containing the desired plasmids (pGADT7 and pGBKT7). Positive protein interactions were assessed on $\mathrm{SD} /$-Trp-Leu-His-Ade medium supplemented with X-a-galactosidase (X- $\alpha$-gal) after being incubated at $28^{\circ} \mathrm{C}$ for four days. For the yeast complementation assay, sensitivity of the different yeast strains to Congo red was determined as described previously ${ }^{28}$. In brief, yeast cells were cultured in YPD or SD-Ura (for strains bearing YEp352 plasmid) medium at $24^{\circ} \mathrm{C}$ overnight., The cells were collected by centrifugation at $5000 \times g$ for $2 \mathrm{~min}$, washed twice with $\mathrm{ddH}_{2} \mathrm{O}$, the cells were diluted to $3 \times 10^{6}$ cells ml ${ }^{-1}$ and then further five 1:5 serial dilutions. Cell suspension (five microliters) were spotted onto YPD medium and YPD supplemented with Congo red for 3 days at $30^{\circ} \mathrm{C}$.

Reporting summary. Further information on research design is available in the Nature Research Reporting Summary linked to this article.

\section{Data availability}

The data supporting the results of this study are available within the article and its Supplementary Information files. The source data underlying Figs. 3a, 4a-f, 5a, 6b, f, 7b, d, f, and $8 \mathrm{~b}, \mathrm{c}$ and Supplementary Figs $5 \mathrm{~b}, 6,7,9 \mathrm{a}-\mathrm{c}, \mathrm{e}, 10$ and 11 are provided as a Source Data file. The authors declare that the other data supporting the findings of this study are available from the corresponding author upon request. Source data are provided with this paper. 
Received: 10 June 2020; Accepted: 10 March 2021;

Published online: 12 April 2021

\section{References}

1. Amselem, J. et al. Genomic analysis of the necrotrophic fungal pathogens Sclerotinia sclerotiorum and Botrytis cinerea. PLoS Genet. 7, e1002230 (2011).

2. Dean, R. et al. The Top 10 fungal pathogens in molecular plant pathology. Mol. Plant Pathol. 13, 414-430 (2012).

3. Eizner, E. et al. Characterization of Botrytis-plant interactions using PathTrack $\odot$ - an automated system for dynamic analysis of disease development. Mol. Plant Pathol. 18, 503-512 (2017).

4. Shlezinger, N. et al. Anti-apoptotic machinery protects the necrotrophic fungus Botrytis cinerea from host-induced apoptotic-like cell death during plant infection. PLoS Pathog. 7, e1002185 (2011).

5. Zhu, W. et al. BcXYG1, a secreted xyloglucanase from Botrytis cinerea, triggers both cell death and plant immune responses. Plant Physiol. 175, 438-456 (2017).

6. Bailey, B. A. Purification of a protein from culture filtrates of Fusarium oxysporum that induces ethylene and necrosis in leaves of Erythroxylum coca. Phytopathology 85, 1250-1255 (1995).

7. Pemberton, C. L. \& Salmond, G. P. The Nep1-like proteins-a growing family of microbial elicitors of plant necrosis. Mol. Plant Pathol. 5, 353-359 (2004).

8. Lenarčič, T. et al. Eudicot plant-specific sphingolipids determine host selectivity of microbial NLP cytolysins. Science 358, 1431-1434 (2017).

9. Lenarčič, T. et al. Molecular basis for functional diversity among microbial Nep1-like proteins. PLoS Pathog. 15, e1007951 (2019).

10. Seidl, M. F. \& Van den Ackerveken, G. Activity and phylogenetics of the broadly occurring family of microbial Nep1-like proteins. Annu. Rev. Phytopathol. 57, 367-386 (2019).

11. At, Have, Mulder, W., Visser, J. \& van Kan, J. A. The endopolygalacturonase gene Bcpg1 is required for full virulence of Botrytis cinerea. Mol. PlantMicrobe Interact. 11, 1009-1016 (1998).

12. Noda, J., Brito, N. \& González, C. The Botrytis cinerea xylanase Xyn11A contributes to virulence with its necrotizing activity, not with its catalytic activity. BMC Plant Biol. 10, 38 (2010).

13. Yang, Y., Yang, X., Dong, Y. \& Qiu, D. The Botrytis cinerea xylanase BcXyll modulates plant immunity. Front. Microbiol. 9, 2535 (2018).

14. Zhang, Y., Zhang, Y., Qiu, D., Zeng, H. \& Guo, L. Yang X. BcGs1, a glycoprotein from Botrytis cinerea, elicits defence response and improves disease resistance in host plants. Biochem. Biophys. Res. Commun. 457, 627-634 (2015).

15. Frías, M., González, M. A., González, C. \& Brito, N. A 25-residue peptide from Botrytis cinerea xylanase BcXyn11A elicits plant defenses. Front. Plant Sci. 10, 474 (2019).

16. Pérez-Hernández, A., González, M., González, C. \& Brito, N. The elicitor protein BcIEB1 and the derived peptide ieb35 provide long-term plant protection. Plant Pathol. 69, 807-817 (2020).

17. Kars, I. et al. Necrotizing activity of five Botrytis cinerea endopolygalacturonases produced in Pichia pastoris. Plant J. 43, 213-225 (2005).

18. Rodríguez-Peña, J. M., Cid, V. J., Arroyo, J. \& Nombela, C. A novel family of cell wall-related proteins regulated differently during the yeast life cycle. Mol. Cell. Biol. 20, 3245-3255 (2000).

19. Pardini, G. et al. The CRH family coding for cell wall glycosylphosphatidylinositol proteins with a predicted transglycosidase domain affects cell wall organization and virulence of Candida albicans. J. Biol. Chem. 281, 40399-40411 (2006).

20. Fang, W. et al. Mechanisms of redundancy and specificity of the Aspergillus fumigatus Crh transglycosylases. Nat. Commun. 10, 1-10 (2019).

21. Blanco-Ulate, B. et al. Genome-wide transcriptional profiling of Botrytis cinerea genes targeting plant cell walls during infections of different hosts. Front. Plant Sci. 5, 435 (2014).

22. González-Fernández, R. et al. Proteomic analysis of mycelium and secretome of different Botrytis cinerea wild-type strains. J. Proteom. 97, 195-221 (2014).

23. Müller, N. et al. Investigations on VELVET regulatory mutants confirm the role of host tissue acidification and secretion of proteins in the pathogenesis of Botrytis cinerea. N. Phytologist 219, 1062-1074 (2018).

24. Schouten, A., Van Baarlen, P. \& Van Kan, J. A. Phytotoxic Nep1-like proteins from the necrotrophic fungus Botrytis cinerea associate with membranes and the nucleus of plant cells. N. Phytologist 177, 493-505 (2008).

25. Frías, M. \& González, C. Brito N. BcSpl1, a cerato-platanin family protein, contributes to Botrytis cinerea virulence and elicits the hypersensitive response in the host. N. Phytologist 192, 483-495 (2011).

26. Fillinger, S. \& Elad, Y. Botrytis: The Fungus, the Pathogen and its Management in Agricultural Systems (Springer, 2016).
27. Chagué, V. et al. Ethylene sensing and gene activation in Botrytis cinerea: a missing link in ethylene regulation of fungus-plant interactions? Mol. Plantmicrobe Interact. 19, 33-42 (2006).

28. Blanco, N. et al. Structural and functional analysis of yeast Crh1 and Crh2 transglycosylases. FEBS J. 282, 715-731 (2015).

29. Hwang, J. S., Seo, D. H. \& Kim, J. Y. Soluble forms of YlCrhlp and YlCrh2p, cell wall proteins of Yarrowia lipolytica, have $\beta$-1, 3-glycosidase activity. Yeast 23, 803-812 (2006).

30. Agrawal, P. et al. CPPsite 2.0: a repository of experimentally validated cellpenetrating peptides. Nucleic Acids Res. 44, D1098-D1103 (2016).

31. Birch, P. R., Rehmany, A. P., Pritchard, L., Kamoun, S. \& Beynon, J. L. Trafficking arms: oomycete effectors enter host plant cells. Trends Microbiol. 14, 8-11 (2006).

32. Whisson, S. C. et al. A translocation signal for delivery of oomycete effector proteins into host plant cells. Nature 450, 115-118 (2007).

33. Presti, L. L. \& Kahmann, R. How filamentous plant pathogen effectors are translocated to host cells. Curr. Opin. plant Biol. 38, 19-24 (2017).

34. Rehmany, A. P. et al. Differential recognition of highly divergent downy mildew avirulence gene alleles by RPP1 resistance genes from two Arabidopsis lines. Plant Cell 17, 1839-1850 (2005).

35. Torto, T. A. et al. EST mining and functional expression assays identify extracellular effector proteins from the plant pathogen Phytophthora. Genome Res. 13, 1675-1685 (2003).

36. Schornack, S. et al. Ancient class of translocated oomycete effectors targets the host nucleus. Proc. Natl Acad. Sci. USA 107, 17421-17426 (2010).

37. Kemen, E. et al. Gene gain and loss during evolution of obligate parasitism in the white rust pathogen of Arabidopsis thaliana. PLoS Biol. 9, e1001094 (2011).

38. Dou, D. et al. RXLR-mediated entry of Phytophthora sojae effector Avrlb into soybean cells does not require pathogen-encoded machinery. Plant Cell 20, 1930-1947 (2008).

39. Kale, S. D. et al. External lipid PI3P mediates entry of eukaryotic pathogen effectors into plant and animal host cells. Cell 142, 284-295 (2010).

40. Plett, J. M. et al. A secreted effector protein of Laccaria bicolor is required for symbiosis development. Curr. Biol. 21, 1197-1203 (2011).

41. Gu, B. et al. Rust secreted protein Ps87 is conserved in diverse fungal pathogens and contains a RXLR-like motif sufficient for translocation into plant cells. PLoS ONE 6, e27217 (2011).

42. Di, X., Gomila, J., Ma, L., van den Burg, H. A. \& Takken, F. L. Uptake of the Fusarium effector Avr2 by tomato is not a cell autonomous event. Front. Plant Sci. 7, 1915 (2016).

43. Cabib, E., Blanco, N., Grau, C. \& Rodríguez-Peña, J. M. Arroyo J. Crhlp and Crh2p are required for the cross-linking of chitin to $\beta$ (1-6) glucan in the Saccharomyces cerevisiae cell wall. Mol. Microbiol. 63, 921-935 (2007).

44. Armenteros, J. J. A. et al. SignalP 5.0 improves signal peptide predictions using deep neural networks. Nat. Biotechnol. 37, 420-423 (2019).

45. Krogh, A., Larsson, B., Von Heijne, G. \& Sonnhammer, E. L. Predicting transmembrane protein topology with a hidden Markov model: application to complete genomes. J. Mol. Biol. 305, 567-580 (2001).

46. Letunic, I. \& Bork, P. 20 years of the SMART protein domain annotation resource. Nucleic Acids Res. 46, D493-D496 (2018).

47. Madeira, F. et al. The EMBL-EBI search and sequence analysis tools APIs in 2019. Nucleic Acids Res. 47, W636-W641 (2019).

48. Zimmermann, L. et al. A completely reimplemented MPI bioinformatics toolkit with a new HHpred server at its core. J. Mol. Biol. 430, 2237-2243 (2018).

49. Ashkenazy, H. et al. ConSurf 2016: an improved methodology to estimate and visualize evolutionary conservation in macromolecules. Nucleic Acids Res. $\mathbf{4 4}$ W344-W350 (2016).

50. Šali, A. \& Blundell, T. L. Comparative protein modelling by satisfaction of spatial restraints. J. Mol. Biol. 234, 779-815 (1993).

51. Lindahl, E., Hess, B. \& Van Der Spoel, D. GROMACS 3.0: a package for molecular simulation and trajectory analysis. Mol. Modeling Annu. 7, 306-317 (2001).

52. Lindorff-Larsen, $\mathrm{K}$. et al. Improved side-chain torsion potentials for the Amber ff99SB protein force field. Proteins: Struct., Funct., Bioinforma. 78, 1950-1958 (2010).

53. Krissinel, E. \& Henrick, K. Inference of macromolecular assemblies from crystalline state. J. Mol. Biol. 372, 774-797 (2007).

54. Yang, G. et al. A cerato-platanin protein SsCP1 targets plant PR1 and contributes to virulence of Sclerotinia sclerotiorum. N. Phytologist 217, 739-755 (2018).

55. Kostylev, M., Otwell, A. E., Richardson, R. E. \& Suzuki, Y. Cloning should be simple: Escherichia coli DH5a-mediated assembly of multiple DNA fragments with short end homologies. PLoS ONE 10, e0137466 (2015).

56. Schumacher, J. Tools for Botrytis cinerea: new expression vectors make the gray mold fungus more accessible to cell biology approaches. Fungal Genet. Biol. 49, 483-497 (2012). 
57. Wang, Q. et al. Transcriptional programming and functional interactions within the Phytophthora sojae RXLR effector repertoire. Plant Cell 23, 2064-2086 (2011).

58. Ma, L., Salas, O., Bowler, K. \& Bar-Peled, M. Sharon A. UDP-4-Keto-6deoxyglucose, a transient antifungal metabolite, weakens the fungal cell wall partly by inhibition of UDP-Galactopyranose Mutase. mBio 8, e01559-01517 (2017).

59. Pizarro, L. et al. Tomato prenylated RAB acceptor protein 1 modulates trafficking and degradation of the pattern recognition receptor LeEIX2, affecting the innate immune response. Front. Plant Sci. 9, 257 (2018).

60. Ma, L. et al. Genetic alteration of UDP-rhamnose metabolism in Botrytis cinerea leads to the accumulation of UDP-KDG that adversely affects development and pathogenicity. Mol. Plant Pathol. 18, 263-275 (2017).

61. Liu, J. K. et al. The key gluconeogenic gene PCK1 is crucial for virulence of Botrytis cinerea via initiating its conidial germination and host penetration. Environ. Microbiol. 20, 1794-1814 (2018).

62. Soulié, M. C. et al. Botrytis cinerea virulence is drastically reduced after disruption of chitin synthase class III gene (Bcchs3a). Cell. Microbiol. 8 , 1310-1321 (2006).

63. Li, Q. et al. A phytophthora capsici effector targets ACD11 binding partners that regulate ROS-mediated defense response in Arabidopsis. Mol. Plant 12, 565-581 (2019).

64. Liu, Z. \& Friesen, T. DAB staining and visualization of hydrogen peroxide in wheat leaves. Bioprotocol 2, e309 (2012).

65. Barhoom, S. \& Sharon, A. cAMP regulation of "pathogenic" and "saprophytic" fungal spore germination. Fungal Genet. Biol. 41, 317-326 (2004).

66. Zhang, X., Henriques, R., Lin, S.-S., Niu, Q.-W. \& Chua, N.-H. Agrobacterium-mediated transformation of Arabidopsis thaliana using the floral dip method. Nat. Protoc. 1, 641 (2006).

67. Gietz, R. D. \& Woods, R. A. Yeast transformation by the LiAc/SS carrier DNA/ PEG method. In Yeast Protocol. Methods Mol. Biol. vol 313. (ed. Xiao, W.) (Humana Press, Totowa, NJ, 2006). https://doi.org/10.1385/1-59259-958-3:107.

\section{Acknowledgements}

We are grateful to Prof. Adi Avni and Mrs. Orian Taig for provision of and assistance with tomato cell cultures. This research was supported by Research Grant Award No. IS4937-16 from BARD, The United States - Israel Binational Agricultural Research and Development Fund to A.S. and grants BIO2016-79289-P and PID2019-105223GB-I00 (Ministerio de Economía y Competitividad, MINECO, Spain) to J.A.

\section{Author contributions}

K.B., L.S., and A.S. designed the experiments, and K.B. and A.S. wrote the paper with inputs from all authors; K.B., L.S., W.Z., N.J., R.F., A.B.S., and W.Z. performed the experiments; T.M., J.A., and G.M. analyzed the data and provided critical feedback.

\section{Competing interests}

The authors declare no competing interests.

\section{Additional information}

Supplementary information The online version contains supplementary material available at https://doi.org/10.1038/s41467-021-22436-1.

Correspondence and requests for materials should be addressed to A.S.

Peer review information Nature Communications thanks Li Dayong, Bruno Moerschbacher and the other, anonymous, reviewers for their contribution to the peer review of this work. Peer reviewer reports are available.

Reprints and permission information is available at http://www.nature.com/reprints

Publisher's note Springer Nature remains neutral with regard to jurisdictional claims in published maps and institutional affiliations.

\begin{abstract}
(c) (i) Open Access This article is licensed under a Creative Commons Attribution 4.0 International License, which permits use, sharing, adaptation, distribution and reproduction in any medium or format, as long as you give appropriate credit to the original author(s) and the source, provide a link to the Creative Commons license, and indicate if changes were made. The images or other third party material in this article are included in the article's Creative Commons license, unless indicated otherwise in a credit line to the material. If material is not included in the article's Creative Commons license and your intended use is not permitted by statutory regulation or exceeds the permitted use, you will need to obtain permission directly from the copyright holder. To view a copy of this license, visit http://creativecommons.org/ licenses/by/4.0/.
\end{abstract}

(C) The Author(s) 2021 